\title{
Holonomy and inverse-triad corrections in spherical models coupled to matter
}

\author{
Asier Alonso-Bardaji ${ }^{\mathrm{a}}{ }^{\mathbb{D}}$, David Brizuela ${ }^{\mathrm{b}}$ \\ Fisika Saila, Universidad del País Vasco/Euskal Herriko Unibertsitatea (UPV/EHU), Barrio Sarriena s/n, 48940 Leioa, Spain
}

Received: 2 December 2020 / Accepted: 19 March 2021 / Published online: 2 April 2021

(C) The Author(s) 2021

\begin{abstract}
Loop quantum gravity introduces two characteristic modifications in the classical constraints of general relativity: the holonomy and inverse-triad corrections. In this paper, a systematic construction of anomaly-free effective constraints encoding such corrections is developed for spherically symmetric spacetimes. The starting point of the analysis is a generic Hamiltonian constraint where free functions of the triad and curvature components as well as non-minimal couplings between geometric and matter degrees of freedom are considered. Then, the requirement of anomaly freedom is imposed in order to obtain a modified Hamiltonian that forms a first-class algebra. In this way, we construct a family of consistent deformations of spherical general relativity, which generalizes previous results in the literature. The discussed derivation is implemented for vacuum as well as for two matter models: dust and scalar field. Nonetheless, only the deformed vacuum model admits free functions of the connection components. Therefore, under the present assumptions, we conclude that holonomy corrections are not allowed in the presence of these matter fields.
\end{abstract}

\section{Introduction}

One of the main aspects a quantum theory of gravity must face is that regarding the singularities of general relativity. The discrete nature of spacetime predicted by loop quantum gravity may provide an answer to this problem [1]. In fact, symmetry-reduced homogeneous models have been widely studied, particularly those related to cosmology, yielding classical singularity resolution via a cosmological bounce [2-4].

However, the extreme energy scales at which quantumgravity phenomena become relevant make experimental evidence still unreachable and predictions of any quantum-

\footnotetext{
a e-mail: asier.alonso@ehu.eus (corresponding author)

b e-mail: david.brizuela@ehu.eus
}

gravity theory seem hard to test. Nevertheless, a general expectation is that these effects leave observable trails at lower energies, which could be studied via effective theories. In fact, in the context of loop quantum cosmology, effective schemes provide an accurate description of the evolution when compared to the full quantum dynamics $[2,3]$. In addition, these effective theories have been widely used to study the interior of spherical black holes, which are described by homogeneous but anisotropic Kantowski-Sachs spaces. The literature presents a wide variety of predictions [5-14]; for instance, the possibility of a quantum transition from a black hole into a white hole, resembling the cosmological bounce.

The usual approach to obtain effective equations is to modify the Hamiltonian in such a way that expected quantum effects from loop quantum gravity are included. Among these modifications, we find two main branches: classical divergences when the volume of a region tends to zero are regularized and encoded in the so-called inverse-triad modifications, whereas holonomy corrections are directly related to the spacetime discreteness predicted by the theory. In homogeneous models, as the diffeomorphism constraint vanishes off-shell, one can include by hand a wide variety of modifications. However, the construction of a consistent effective theory becomes rather challenging when non-homogeneous scenarios are considered. In these cases, the diffeomorphism constraint is no longer vanishing and one finds that the quantized notion of spacetime collides with the continuous diffeomorphism symmetry of general relativity.

Several different attempts to study inhomogeneous models can be found in the literature. For instance, Gowdy models have been analyzed by means of the procedure named hybrid quantization [15]. This approach splits the problem in two: a Bianchi I model describing the homogeneous background spacetime, quantized following the loop quantum cosmology program, and a Fock quantization of the remaining inhomogeneities. This scheme has also been applied to cosmological perturbations [16-19]. In the same context, one must also 
mention the dressed-metric approach [20-22]. Both methods make similar assumptions and provide a completely quantized theory to analyze cosmological perturbations. However, it is not clear whether these frameworks respect the general covariance of the theory [23]. In fact, this is the main guideline of the consistent constraint deformation approach. This alternative formalism constructs effective theories demanding that the modified constraints form an anomaly-free algebra. References [24-29] implement this approach for cosmological perturbations.

Spherically symmetric models are of particular relevance, as they would be a first step to describe black holes and gravitational collapse. In this context, it was found that the Abelianization of the constraints could lead to a consistent quantization [30], even under the presence of a scalar matter field [31]. Nonetheless, this approach suffers equally from the lack of covariance, as analyzed in [32]. On the other hand, the consistent constraint deformation approach has already been applied to several spherical models, both in vacuum and coupled to different matter fields [32-38], leading to promising results. In particular, the effective line element corresponding to the holonomy-corrected constraints might present a signature change when approaching the classical singularity. From this standpoint, some interior region of the black hole would be Euclidean, and the usual notions of causality and time evolution would not apply. In particular, in [39], it is argued that this prediction rules out the possibility of bouncing black holes. Unfortunately, most studies based on this methodology acknowledge that holonomy modifications can only be implemented in vacuum. The requirement of anomaly freedom in the presence of matter with local degrees of freedom restricts even more feasible modifications, leaving no room for holonomy corrections [37].

Therefore, the goal of this paper is to perform a systematic analysis of possible modifications to classical constraints of general relativity in spherical symmetry. We will consider the vacuum case as well as gravity coupled to two different matter models: dust and scalar field. A key difference between the present and previous studies will be the possibility for nonminimal couplings between matter and geometric degrees of freedom as well as for polymerization of the matter variables. Nonetheless, in agreement with previous works, we will find that only the vacuum model admits holonomy corrections.

The rest of the paper is organized as follows. Section 2 includes a brief summary of the constraint structure of classical general relativity (2.1) and introduces the main features of inverse-triad and holonomy corrections (2.2). In Sect. 3, we compute the Poisson brackets between modified constraints, assuming a completely general kinetic part in the Hamiltonian for the vacuum model. In Sects. 4.1 and 4.2, respectively, we will extend our analysis to include a scalar field and dust with possible non-minimal couplings between matter and geometry. Finally, Sect. 5 summarizes the main results and presents the conclusions of our study.

\section{Constraint algebra}

\subsection{Classical theory}

The canonical formalism of general relativity shows that this theory is a completely constrained dynamical system. The total Hamiltonian is a linear combination of the so-called Hamiltonian constraint and diffeomorphism constraint, with the coefficients given by the lapse function $(N)$ and the shift vector, respectively. Since we will be assuming spherical symmetry, the angular components of the diffeomorphism constraint will vanish off-shell and the only relevant part of the shift vector will be its radial component $\left(N^{r}\right)$.

Following the usual approach in loop quantum gravity, the basic variables will be the $U(1)$-invariant components of the spherically symmetric triad, $E^{r}$ and $E^{\varphi}$. Their canonically conjugate momenta will be given by the elements of the Ashtekar $s u$ (2) connection, which can be written in terms of the extrinsic curvature components $K_{r}$ and $K_{\varphi}$. Setting the Newton constant to one, $G=1$, the symplectic structure of the phase space is given by the Poisson brackets,

$\left\{K_{r}(x), E^{r}(y)\right\}=\left\{K_{\varphi}(x), E^{\varphi}(y)\right\}=\delta(x-y)$.

In terms of these variables, the vacuum Hamiltonian and diffeomorphism constraints of classical general relativity read respectively as follows,

$\mathcal{H}_{g}:=\sqrt{E^{r}}\left(\Gamma_{\varphi}^{\prime}-2 K_{r} K_{\varphi}\right)-\frac{E^{\varphi}}{2 \sqrt{E^{r}}}\left(1+K_{\varphi}^{2}-\Gamma_{\varphi}^{2}\right)$,

$\mathcal{D}_{g}:=\left(K_{\varphi}\right)^{\prime} E^{\varphi}-\left(E^{r}\right)^{\prime} K_{r}$,

where $\Gamma_{\varphi}:=\left(E^{r}\right)^{\prime} /\left(2 E^{\varphi}\right)$ is the angular component of the spin connection.

In the canonical formalism, the covariance of general relativity is not explicit, but it can be read from the hypersurface deformation algebra,

$$
\begin{aligned}
\left\{D\left[N_{1}^{r}\right], D\left[N_{2}^{r}\right]\right\} & =D\left[N_{1}^{r} N_{2}^{r^{\prime}}-N_{1}^{r^{\prime}} N_{2}^{r}\right], \\
\left\{D\left[N^{r}\right], H[N]\right\} & =H\left[N^{r} N^{\prime}\right], \\
\left\{H\left[N_{1}\right], H\left[N_{2}\right]\right\} & =D\left[q^{r r}\left(N_{1} N_{2}^{\prime}-N_{1}^{\prime} N_{2}\right)\right],
\end{aligned}
$$

where the smeared form of the constraints has been introduced as $D\left[N^{r}\right]:=\int d r N^{r} \mathcal{D}$ and $H[N]:=\int d r N \mathcal{H}$, and the prime denotes the derivative with respect to the radial variable $r$. Note that, in the last bracket, the component $q^{r r}$ of the inverse three-metric appears, which can be obtained from the spherically symmetric spatial line element, 
$d \sigma^{2}=\frac{\left(E^{\varphi}\right)^{2}}{E^{r}} d r^{2}+E^{r} d \Omega^{2}$

\subsection{Corrections motivated by loop quantum gravity}

In this section, the two different types of corrections motivated by the theory of loop quantum gravity are reviewed: the so-called inverse-triad and holonomy corrections.

\subsubsection{Inverse-triad corrections}

In loop quantum gravity, the operators built from triad components contain zero in their discrete spectra. Therefore, they cannot be inverted. Nonetheless, a regularization procedure is followed to obtain well-defined operators [40,41]. The expectation values of these regularized operators mimic the classical behavior at large scales but present deviations from classical divergences at small volumes. In the context of effective theories, inverse-triad corrections are introduced to account for the regularization carried out in the full theory.

In principle, only terms with explicit triad terms in the denominator should be held to corrections. However, in the symmetry reduction process that leads to expression (2), some triad factors coming from the volume element have been simplified. At this level, we will not discuss the implications of correcting just the volume element or individual terms in the Hamiltonian. We will introduce two independent multiplicative functions, $\alpha_{1}$ and $\alpha_{2}$,

$$
\begin{aligned}
\mathcal{H}_{g}^{(t)}:= & \alpha_{1}\left(E^{r}\right) \sqrt{E^{r}}\left(\Gamma_{\varphi}^{\prime}-2 K_{r} K_{\varphi}\right) \\
& -\alpha_{2}\left(E^{r}\right) \frac{E^{\varphi}}{2 \sqrt{E^{r}}}\left(1+K_{\varphi}^{2}-\Gamma_{\varphi}^{2}\right),
\end{aligned}
$$

so that when both tend to one, we recover the classical Hamiltonian constraint (2). If the origin of the corrections was the volume element, then both correction functions would be equal $\alpha_{1}=\alpha_{2}$.

One can check that this deformed Hamiltonian and the classical diffeomorphism constraint (3) do close algebra. More precisely, the algebraic relations (4a) and (4b) remain unmodified, but the third bracket (4c) acquires a deformation given by the function $\alpha_{1}$ :

$$
\left\{H_{g}^{(t)}\left[N_{1}\right], H_{g}^{(t)}\left[N_{2}\right]\right\}=D_{g}\left[\alpha_{1}^{2} q^{r r}\left(N_{1} N_{2}^{\prime}-N_{1}^{\prime} N_{2}\right)\right],
$$

where $H_{g}^{(t)}[N]$ is the smeared form of $\mathcal{H}_{g}^{(t)}$. Note that if only terms with explicit powers of inverse triads were modified in (2), $\alpha_{1}$ would be one and the whole algebraic structure would remain undeformed.

\subsubsection{Holonomy modifications}

The operator associated to the connection is not well defined in loop quantum gravity. Alternatively, one considers holonomies, the exponential form of parallel-transported connections, which do have a definite operator counterpart. In order to construct effective theories, the general idea is that connection components should be replaced with periodic functions. This procedure is sometimes referred to as polymerization.

In the present approach, holonomy corrections will involve the replacement of the extrinsic curvature variables by generic functions. The requisite of a closed algebra will impose conditions over these modifications.

For instance, following the standard procedure found in the literature [32,34,35], let us replace the terms $K_{\varphi}$ and $K_{\varphi}^{2}$ in expression (2) by $f_{1}\left(K_{\varphi}\right)$ and $f_{2}\left(K_{\varphi}\right)$, respectively, and define the holonomy-modified Hamiltonian constraint,

$\mathcal{H}_{g}^{(h)}:=\sqrt{E^{r}}\left(\Gamma_{\varphi}^{\prime}-2 K_{r} f_{1}\right)-\frac{E^{\varphi}}{2 \sqrt{E^{r}}}\left(1+f_{2}-\Gamma_{\varphi}^{2}\right)$,

along with its smeared version $H_{g}^{(h)}[N]:=\int d r N \mathcal{H}_{g}^{(h)}$.

Considering this deformed Hamiltonian in combination with the classical diffeomorphism constraint (3), one can check that the first two brackets (4a) and (4b) of the hypersurface deformation algebra remain unmodified, while the third one generates an anomalous term:

$$
\begin{gathered}
\left\{H_{g}^{(h)}\left[N_{1}\right], H_{g}^{(h)}\left[N_{2}\right]\right\}=D_{g}\left[\frac{\partial f_{1}}{\partial K_{\varphi}} q^{r r}\left(N_{1} N_{2}^{\prime}-N_{1}^{\prime} N_{2}\right)\right] \\
+\frac{\left(E^{r}\right)^{\prime}}{4 E^{\varphi}}\left(N_{1} N_{2}^{\prime}-N_{1}^{\prime} N_{2}\right)\left(2 f_{1}-\frac{\partial f_{2}}{\partial K_{\varphi}}\right) .
\end{gathered}
$$

In order to cancel the anomaly, one needs to enforce the relation $2 f_{1}=\partial f_{2} / \partial K_{\varphi}$. Thus, both functions are not independent and, following the motivation mentioned above, they are usually chosen to have a sinusoidal form. For example, $f_{1}=\sin \left(\mu K_{\varphi}\right) / \mu$, where the parameter $\mu$ is assumed to be related to the minimum area predicted by the theory. In the limit $\mu \rightarrow 0$, the Hamiltonian (8) recovers its classical form (2).

Finally, let us emphasize that the two types of corrections introduced in this section are compatible. That is, one can consider the deformed Hamiltonian constraint,

$$
\begin{aligned}
\mathcal{H}_{g}^{(h t)}:= & \alpha_{1}\left(E^{r}\right) \sqrt{E^{r}}\left(\Gamma_{\varphi}^{\prime}-2 K_{r} \frac{\partial f\left(K_{\varphi}\right)}{\partial K_{\varphi}}\right) \\
& -\alpha_{2}\left(E^{r}\right) \frac{E^{\varphi}}{2 \sqrt{E^{r}}}\left(1+2 f\left(K_{\varphi}\right)-\Gamma_{\varphi}^{2}\right),
\end{aligned}
$$

with generic functions $\alpha_{1}, \alpha_{2}$ and $f$. This Hamiltonian closes algebra along with the classical diffeomorphism constraint (3). The brackets (4a) and (4b) remain unchanged, whereas 
the one between modified Hamiltonian constraints presents a deformation equal to the product of corrections in the righthand sides of (7) and (9):

$$
\begin{aligned}
& \left\{H_{g}^{(h t)}\left[N_{1}\right], H_{g}^{(h t)}\left[N_{2}\right]\right\} \\
& \quad=D_{g}\left[\alpha_{1}^{2} \frac{\partial^{2} f}{\partial K_{\varphi}^{2}} q^{r r}\left(N_{1} N_{2}^{\prime}-N_{1}^{\prime} N_{2}\right)\right],
\end{aligned}
$$

with the smeared form $H_{g}^{(h t)}:=\int d r N \mathcal{H}_{g}^{(h t)}$.

\section{Vacuum}

Before studying the possibility of constructing effective theories with inverse-triad and holonomy corrections in the presence of matter fields, let us first try to generalize the known results for vacuum summarized in the previous section. For such a purpose, we will consider the following deformed Hamiltonian constraint,

$$
\begin{aligned}
\widetilde{\mathcal{H}}_{g}= & f_{g}\left(E^{r}, E^{\varphi}, K_{r}, K_{\varphi}\right)+\eta_{1}\left(E^{r}, E^{\varphi}\right) \sqrt{E^{r}} \Gamma_{\varphi}^{\prime} \\
& -\eta_{2}\left(E^{r}, E^{\varphi}\right) \frac{E^{\varphi}}{2 \sqrt{E^{r}}}+\eta_{3}\left(E^{r}, E^{\varphi}\right) \frac{E^{\varphi} \Gamma_{\varphi}^{2}}{2 \sqrt{E^{r}}} .
\end{aligned}
$$

Note that the usual kinetic part (all terms with an explicit factor of $K_{r}$ or $K_{\varphi}$ ) of the classical Hamiltonian constraint (2) has been replaced with a generic function $f_{g}$, which is assumed to depend on all canonical variables. This function will encode the possible holonomy corrections of the model as well as inverse-triad modifications. In this way, the holonomy corrections might also depend on triad components, as in the improved-dynamics scheme of loop quantum cosmology [42]. Additionally, we have included three free functions $\eta_{1}, \eta_{2}$, and $\eta_{3}$, with dependence on both triad components $E^{r}$ and $E^{\varphi}$, in front of each remaining term, to account for additional inverse-triad corrections. In fact, we could expand the derivative of the spin-connection $\Gamma_{\varphi}^{\prime}$ in two terms depending on the derivatives of the triad components and include a correction multiplying each of them. Instead of just one function $\left(\eta_{1}\right)$, this would lead to two independent corrections but with the same final results, since anomaly freedom would demand these two functions to be equal. Therefore, with no loss of generality, we will just consider the correction function $\eta_{1}$.

Concerning the diffeomorphism constraint, in this section we will keep it unmodified and its classical form, given in (3), will be considered. The full theory of loop quantum gravity does not modify the diffeomorphism constraint and thus one does not expect it to be modified at an effective level. However, in Appendix A, we will consider generic deformations of the diffeomorphism constraint, along with the form (12) for the Hamiltonian. Following the same methodology as in this section, we will explicitly show that (up to canonical transformations) only a global rescaling of the diffeomorphism constraint is allowed, leading to the same modified Hamiltonian that will be obtained in this section (expression (28) below). Results in Appendix A generalize previous studies that point out that the requirement of anomaly freedom only admits trivial modifications of the diffeomorphism constraint [38].

Regarding the constraint algebra, it is clear that, since here we did not change the diffeomorphism constraint, relation (4a) remains unmodified. On the other hand, the bracket between the classical diffeomorphism (3) and the modified Hamiltonian constraints (12) gives rise, up to irrelevant global factors, to the following anomalous term,

$$
\begin{aligned}
\mathcal{A}_{g}^{D H}:= & 8 \sqrt{E^{r}}\left[f_{g}-K_{r} \frac{\partial f_{g}}{\partial K_{r}}-\eta_{1} E^{\varphi} \frac{\partial\left(f_{g} / \eta_{1}\right)}{\partial E^{\varphi}}\right] \\
& -4 \eta_{1}\left(E^{\varphi}\right)^{2} \frac{\partial\left(\eta_{2} / \eta_{1}\right)}{\partial E^{\varphi}}+\eta_{1}\left[\left(E^{r}\right)^{\prime}\right]^{2} \frac{\partial\left(\eta_{3} / \eta_{1}\right)}{\partial E^{\varphi}}
\end{aligned}
$$

whereas the bracket of the modified Hamiltonian with itself leads to the anomaly:

$$
\begin{aligned}
\mathcal{A}_{g}^{H H}:= & \left(E^{r}\right)^{\prime}\left[\left(\eta_{1}-\eta_{3}\right) \frac{E^{\varphi}}{2} \frac{\partial f_{g}}{\partial K_{r}}-\eta_{1}^{2} E^{r} E^{\varphi} \frac{\partial^{2}\left(f_{g} / \eta_{1}\right)}{\partial K_{r} \partial E^{r}}\right. \\
& \left.+\eta_{1} E^{r}\left(\frac{\partial f_{g}}{\partial K_{\varphi}}-K_{r} \frac{\partial^{2} f_{g}}{\partial K_{r} \partial K_{\varphi}}\right)\right] \\
& +\left(E^{\varphi}\right)^{\prime} E^{r} E^{\varphi} \eta_{1}^{2} \frac{\partial^{2}\left(f_{g} / \eta_{1}\right)}{\partial E^{\varphi} \partial K_{r}}-\left(K_{r}\right)^{\prime} E^{r} E^{\varphi} \eta_{1} \frac{\partial^{2} f_{g}}{\partial K_{r}^{2}} .
\end{aligned}
$$

Therefore, the condition that the constraints $\widetilde{\mathcal{H}}_{g}$ and $\mathcal{D}_{g}$ form a closed algebra is translated to the vanishing of all the above terms:

$0=\mathcal{A}_{g}^{D H}$,

$0=\mathcal{A}_{g}^{H H}$.

Up to this point, there is some ambiguity in the generic functions defined in the modified constraint. In particular, the third term of (12), the one that goes with the $\eta_{2}$ function, could have been absorbed in the definition of $f_{g}$ as it does not depend on the radial derivatives of the triad components. This means that, with no loss of generality, $\eta_{2}$ can be chosen as desired. Therefore, in order to cancel the last term of the anomaly (13), we will demand,

$\eta_{2}\left(E^{r}, E^{\varphi}\right)=\xi_{2}\left(E^{r}\right) \eta_{1}\left(E^{r}, E^{\varphi}\right) / \xi_{1}\left(E^{r}\right)$,

$\xi_{1}$ and $\xi_{2}$ being free functions of the radial component of the triad. Notice that the generic functions, and $\eta_{1}$ in particular, are assumed to be non-vanishing. 
None of the free functions that are contained in the anomaly equations depend on radial derivatives. Hence, each coefficient of a primed variable must vanish independently and the following five equations, alongside (17), are equivalent to the system (15) and (16):

$$
\begin{aligned}
0= & \frac{\partial\left(\eta_{3} / \eta_{1}\right)}{\partial E^{\varphi}}, \\
0= & f_{g}-K_{r} \frac{\partial f_{g}}{\partial K_{r}}-\eta_{1} E^{\varphi} \frac{\partial\left(f_{g} / \eta_{1}\right)}{\partial E^{\varphi}}, \\
0= & \frac{\partial^{2}\left(f_{g} / \eta_{1}\right)}{\partial E^{\varphi} \partial K_{r}}, \\
0= & \left(\eta_{1}-\eta_{3}\right) \frac{E^{\varphi}}{2} \frac{\partial f_{g}}{\partial K_{r}}-\eta_{1}^{2} E^{r} E^{\varphi} \frac{\partial^{2}\left(f_{g} / \eta_{1}\right)}{\partial K_{r} \partial E^{r}} \\
& +\eta_{1} E^{r}\left(\frac{\partial f_{g}}{\partial K_{\varphi}}-K_{r} \frac{\partial^{2} f_{g}}{\partial K_{r} \partial K_{\varphi}}\right), \\
0= & \frac{\partial^{2} f_{g}}{\partial K_{r}^{2}} .
\end{aligned}
$$

The general solution of Eq. (18) provides the following relation between the functions $\eta_{1}$ and $\eta_{3}$,

$\eta_{3}\left(E^{r}, E^{\varphi}\right)=\xi_{3}\left(E^{r}\right) \eta_{1}\left(E^{r}, E^{\varphi}\right) / \xi_{1}\left(E^{r}\right)$,

with $\xi_{3}$ a free function of the radial component of the triad. In addition, direct integration of Eqs. (20) and (22) leads to the following form for the kinetic term of the Hamiltonian:

$f_{g}=\frac{\eta_{1}\left(E^{r}, E^{\varphi}\right)}{\xi_{1}\left(E^{r}\right)}\left[K_{r} f_{1}\left(E^{r}, K_{\varphi}\right)+f_{2}\left(E^{r}, E^{\varphi}, K_{\varphi}\right)\right]$,

where we have introduced two integration functions $f_{1}$ and $f_{2}$ along with their corresponding dependencies. In particular, note that the dependence on the radial component of the connection, $K_{r}$ is already explicit and, therefore, holonomy corrections depending on $K_{r}$ will not be allowed: the Hamiltonian must be linear in $K_{r}$.

Replacing now (24) in (19), one obtains the relation,

$f_{2}-E^{\varphi} \frac{\partial f_{2}}{\partial E^{\varphi}}=0$,

which implies that $f_{2}$ must be linear in $E^{\varphi}$. In summary, the expression

$f_{g}=-\frac{\eta_{1}}{\xi_{1}}\left[2 \sqrt{E^{r}} K_{r} h_{1}\left(E^{r}, K_{\varphi}\right)+\frac{E^{\varphi}}{2 \sqrt{E^{r}}} h_{2}\left(E^{r}, K_{\varphi}\right)\right]$,

provides the most general function $f_{g}$, that obeys all conditions above. For convenience, we have included some explicit numerical factors and $\sqrt{E^{r}}$ terms, so that this function resembles the classical form of the kinetic part of the constraint when functions $h_{1}$ and $h_{2}$ take the values $K_{\varphi}$ and $K_{\varphi}^{2}$, respectively.

In order to obtain an anomaly-free algebra, the only condition left is given by equation (21). Inserting the forms (23) and (26) in that relation, the following differential equation is obtained:

$\frac{1}{2} \frac{\partial h_{2}}{\partial K_{\varphi}}=\left(\xi_{3}-2 E^{r} \frac{\partial \xi_{1}}{\partial E^{r}}\right) \frac{h_{1}}{\xi_{1}}+2 E^{r} \frac{\partial h_{1}}{\partial E^{r}}$.

Collecting all the conditions we found for the correction functions, the deformed Hamiltonian constraint reads,

$$
\begin{aligned}
\tilde{\mathcal{H}}_{g}= & \eta\left(E^{r}, E^{\varphi}\right)\left[\sqrt{E^{r}}\left(\xi_{1}\left(E^{r}\right) \Gamma_{\varphi}^{\prime}-2 K_{r} h_{1}\left(E^{r}, K_{\varphi}\right)\right)\right. \\
& \left.-\frac{E^{\varphi}}{2 \sqrt{E^{r}}}\left(\xi_{2}\left(E^{r}\right)+h_{2}\left(E^{r}, K_{\varphi}\right)-\xi_{3}\left(E^{r}\right) \Gamma_{\varphi}^{2}\right)\right],
\end{aligned}
$$

where we have defined the free global factor $\eta:=\eta_{1} / \xi_{1}$. Recall that all the modifications are not independent since the additional relation (27) must be obeyed. The classical Hamiltonian constraint is recovered when $\eta=\xi_{1}=\xi_{2}=\xi_{3}=1$, $h_{1}=K_{\varphi}$ and $h_{2}=K_{\varphi}^{2}$, which automatically satisfies the mentioned requisite.

Regarding the physical interpretation of the modifications, functions $\xi_{1}, \xi_{2}$ and $\xi_{3}$ can be interpreted as inverse-triad corrections, whereas $h_{1}$ and $h_{2}$ would represent holonomy modifications. Since these last two have an arbitrary dependence on the radial component of the triad, they could also encode certain inverse-triad corrections. The factor $\eta$ is a trivial modification in the sense that one can always rescale the constraint by any global multiplicative function and the new rescaled constraint will provide an anomaly-free algebra. In addition, this global factor would not have any effect in local dynamics and might only alter global properties of the spacetime. Furthermore, since the Hamiltonian must be a scalar density, the global factor $\eta$ should be of weight zero. This would rule out the dependence of $\eta$ on the angular component of the triad $E^{\varphi}$, for it being a scalar density. Nonetheless, in order to keep the discussion as general as possible, this dependence will be kept so that by incorporating additional densities a scalar object may be formed.

The modified Hamiltonian constraint (28) is the main result of this section and provides a generalization of the already present results in the literature. In particular, expression (10) may be recovered just taking $\eta=1, \xi_{1}=\alpha_{1}$, $\xi_{2}=\xi_{3}=\alpha_{2}, h_{2}=2 \alpha_{2} f$ and $h_{1}=\alpha_{1} \partial f / \partial K_{\varphi}$. Note that, for these choices, relation (27) is automatically satisfied.

The anomaly-free first-class algebra formed by this modified Hamiltonian and the classical diffeomorphism constraint is: 
$\left\{D_{g}\left[N^{r}\right], \widetilde{H}_{g}[N]\right\}=\widetilde{H}_{g}\left[N^{r} N^{\prime}-\left(N^{r}\right)^{\prime} N \frac{E^{\varphi}}{\eta} \frac{\partial \eta}{\partial E^{\varphi}}\right]$,

$\left\{\widetilde{H}_{g}\left[N_{1}\right], \widetilde{H}_{g}\left[N_{2}\right]\right\}=D_{g}\left[\eta^{2} \xi_{1} \frac{\partial h_{1}}{\partial K_{\varphi}} q^{r r}\left(N_{1} N_{2}^{\prime}-N_{1}^{\prime} N_{2}\right)\right]$,

where $\widetilde{H}_{g}[N]$ is the smeared version of $\widetilde{\mathcal{H}}_{g}$. The first bracket picks up corrections from the global factor $\eta$. Nonetheless, the deformation of the bracket (30) might contain relevant information about quantum-gravity effects as one approaches the deep quantum regime, such as signature change [37,39]. Only for the case $\eta^{2} \xi_{1} h_{1}=K_{\varphi}+g\left(E^{r}\right)$, which would imply that $\eta$ is independent of $E^{\varphi}$, one would recover the exact algebra of general relativity.

The procedure we followed imposes the quite restrictive form (28) even if we started from a completely generic kinetic term in the Hamiltonian (12), yielding some interesting features. First, we see that the only correction that depends on the angular component of the triad $E^{\varphi}$ is contained in the global factor $\eta$. Second, there are no free dependences on the radial component of the connection $K_{r}$, which appear in the same way as in the classical Hamiltonian. Therefore, no holonomy modifications dependent on $K_{r}$ are allowed.

The only possible holonomy corrections are encoded in $h_{1}$ and $h_{2}$, which are allowed to be scale-dependent through the radial component of the triad $E^{r}$. This is an interesting aspect of the model as it allows for some kind of improveddynamics scheme, similar to the one considered in the context of loop quantum cosmology [42]. In fact, there are already some examples in the literature with this kind of scaledependent holonomy corrections. For instance, the anomaly equation (27) can be directly compared with results presented in $[32,35]$ (which do not include the additional inverse-triad corrections $\left.\xi_{1}, \xi_{2}, \xi_{3}\right)$. Finally, in Appendix B we analyze possible solutions for $h_{1}$ and $h_{2}$ that satisfy equation (27), assuming a linear expansion of the functions and focusing on leading order terms.

\section{Singular solutions}

For the sake of completeness, we would like to point out that, in order to write and solve the system of equations (15) and (16), all correction functions have been assumed to be non-vanishing. However, it is possible to find two families of singular solutions (in the sense that they are not contained in the previous general case) that involve the vanishing of $\eta_{1}$ :

(i) $\eta_{1}=0$ and $f_{g}=f_{g}\left(E^{r}, E^{\varphi}, K_{\varphi}\right)$.

(ii) $\eta_{1}=0$ and $\eta_{3}=0$.

Each of these modifications in the Hamiltonian provides a first-class algebra, without any further requirement on the remaining functions. Note that one can not directly substitute these values in the expressions above as $\eta_{1}$ appears dividing in the anomalous term (13). In order to check that these are indeed consistent deformations, one needs to compute again the Poisson bracket (29).

Once $\eta_{1}=0$ is chosen, the only derivative term in the deformed Hamiltonian is $\left(E^{r}\right)^{\prime}$. Next, one can consider either (i), to eliminate its canonical conjugate variable $K_{r}$, or (ii), to remove all derivative terms in the Hamiltonian. The latter case corresponds to the homogeneous limit of the model which, following the Belinski-Khalatnikov-Lifshitz (BKL) conjecture [43], is expected to be relevant near the classical singularity.

In contrast to the general case analyzed above, these singular solutions present a less constrained dependence on the connection variables. On the one hand, in (i), although the dependence on $K_{r}$ is fixed, we find a complete freedom regarding $K_{\varphi}$. On the other hand, case (ii) brings complete freedom when choosing the dependence of the Hamiltonian constraint on both connection components, $K_{r}$ and $K_{\varphi}$, allowing for any kind of holonomy correction.

\section{Matter fields}

The complexity of studying holonomy modifications in the presence of matter is widely recognized. In fact, there is a quite general argument that shows the impossibility of consistently including holonomy corrections in the effective Hamiltonian [32]. Let us consider a minimally-coupled matter model with a possibly deformed Hamiltonian constraint $\widetilde{H}_{m}[N]$. The full smeared Hamiltonian is $\widetilde{H}[N]=$ $\widetilde{H}_{g}[N]+\widetilde{H}_{m}[N]$, with the vacuum Hamiltonian $\widetilde{H}_{g}[N]$ given by the smeared form of (28). In order to define a first-class algebra, the bracket

$$
\left\{\widetilde{H}_{g}\left[N_{1}\right]+\widetilde{H}_{m}\left[N_{1}\right], \widetilde{H}_{g}\left[N_{2}\right]+\widetilde{H}_{m}\left[N_{2}\right]\right\},
$$

should be expressed as a linear combination of the full constraints $\widetilde{H}[N]$ and $D\left[N^{r}\right]:=D_{g}\left[N^{r}\right]+D_{m}\left[N^{r}\right], D_{m}\left[N^{r}\right]$ being the matter contribution to the diffeomorphism constraint. It is easy to check that, when the modified matter Hamiltonian $\widetilde{H}_{m}[N]$ depends only on matter variables (including their radial derivatives) and triad components, but it does not depend on curvature components nor radial derivatives of the triad, the sum of the cross brackets,

$\left\{\widetilde{H}_{g}\left[N_{1}\right], \widetilde{H}_{m}\left[N_{2}\right]\right\}+\left\{\widetilde{H}_{m}\left[N_{1}\right], \widetilde{H}_{g}\left[N_{2}\right]\right\}$,

is vanishing due to antisymmetry. As there are no derivatives of $K_{r}$ or $K_{\varphi}$ in the gravitational Hamiltonian and we are assuming no derivatives of the triad components in $H_{m}$, one does not need to perform any integration by parts when com- 
puting these Poisson brackets and, thus, both are proportional to the product $N_{1} N_{2}$.

Now, since the bracket between two vacuum Hamiltonians is proportional to the vacuum diffeomorphism constraint (30), anomaly-freedom demands that the bracket between matter Hamiltonians is proportional to the matter diffeomorphism constraint. In such a case, (31) would read as follows,

$$
\begin{aligned}
& \left\{\widetilde{H}_{g}\left[N_{1}\right]+\widetilde{H}_{m}\left[N_{1}\right], \widetilde{H}_{g}\left[N_{2}\right]+\widetilde{H}_{m}\left[N_{2}\right]\right\} \\
& =\int d r q^{r r}\left(N_{1} N_{2}^{\prime}-N_{1}^{\prime} N_{2}\right)\left[\eta_{1}^{2} \frac{\partial h_{1}}{\partial K_{\varphi}} \mathcal{D}_{g}+\Delta_{m} \mathcal{D}_{m}\right],
\end{aligned}
$$

where, because of our assumptions, $\Delta_{m}$ depends on triad components and matter variables, but not on curvature components $K_{r}$ and $K_{\varphi}$. In order to obtain a closed algebra, $\Delta_{m}$ should be equal to $\eta_{1}^{2} \partial h_{1} / \partial K_{\varphi}$, imposing that $h_{1}$ does not depend on $K_{\varphi}$ and thus ruling out the holonomy corrections that were allowed in vacuum.

This general argument applies to simple matter models, such as minimally coupled scalar fields or dust. Nonetheless, one can try to bypass it by considering more generic relations. In fact, it might well happen that matter and geometric degrees of freedom develop non-minimal couplings as one approaches the quantum regime. In addition, one could expect polymerization, usually carried out only for the geometric degrees of freedom, to affect some of the matter variables, as proposed for instance in [44-46].

The object of this section is to study two different matter models, scalar field and dust, with a quite generic form of the deformed Hamiltonian. In particular, we will allow for non-minimal couplings as well as possible polymerization of both, geometric and matter degrees of freedom. More precisely, we will be assuming a general kinetic term for the modified Hamiltonian constraint, just as in the previous section. This generic term will be allowed to depend on any variable of the model and, thus, any coupling between the different matter and geometric variables will be possible. Furthermore, this general function might also take into account possible polymerization processes for all the degrees of freedom: not only for the curvature terms $K_{r}$ and $K_{\varphi}$, but also for matter variables. In addition, multiplicative corrections depending on triad components and the corresponding matter field will be included on each of the remaining terms of the Hamiltonian, describing in that way possible inverse-triad corrections. At the same time, these functions will enable a more general coupling between matter and geometry.

\subsection{Scalar field}

Assuming spherical symmetry, the contribution to the Hamiltonian and diffeomorphism constraints of a minimally coupled scalar field, $\phi$, are respectively given by,

$$
\begin{aligned}
\mathcal{H}_{\phi} & =\frac{P_{\phi}^{2}}{2 \sqrt{E^{r}} E^{\varphi}}+\frac{\left(E^{r}\right)^{3 / 2}}{2 E^{\varphi}}\left(\phi^{\prime}\right)^{2}+\sqrt{E^{r}} E^{\varphi} V(\phi), \\
\mathcal{D}_{\phi} & =\phi^{\prime} P_{\phi}
\end{aligned}
$$

The momentum $P_{\phi}$ is canonically conjugate to $\phi$, with the Poisson bracket,

$\left\{\phi(x), P_{\phi}(y)\right\}=\delta(x-y)$,

that completes the phase-space structure given in (1).

As in Sect. 3, we will leave the diffeomorphism constraint unmodified, $\mathcal{D}=\mathcal{D}_{g}+\mathcal{D}_{\phi}$ and will introduce the following initial proposal for the deformed Hamiltonian constraint:

$$
\begin{aligned}
\widetilde{\mathcal{H}}= & f\left(E^{r}, E^{\varphi}, \phi, K_{r}, K_{\varphi}, P_{\phi}\right)+\eta_{1}\left(E^{r}, E^{\varphi}, \phi\right) \sqrt{E^{r}} \Gamma_{\varphi}^{\prime} \\
& -\eta_{2}\left(E^{r}, E^{\varphi}, \phi\right) \frac{E^{\varphi}}{2 \sqrt{E^{r}}}+\eta_{3}\left(E^{r}, E^{\varphi}, \phi\right) \frac{E^{\varphi} \Gamma_{\varphi}^{2}}{2 \sqrt{E^{r}}} \\
& +\eta_{4}\left(E^{r}, E^{\varphi}, \phi\right) \frac{\left(E^{r}\right)^{3 / 2}}{2 E^{\varphi}}\left(\phi^{\prime}\right)^{2}
\end{aligned}
$$

The generic function $f$ of the deformed Hamiltonian is allowed to depend on all the canonical variables but not on their radial derivatives. It accounts for generic couplings between different variables, as well as inverse-triad and holonomy corrections (in particular, it encodes a possible polymerization of the matter variables). In addition, the free multiplicative functions that appear in the remaining terms stand for possible inverse-triad corrections and allow the scalar field to couple to radial derivatives of the triad.

If one computes the Poisson brackets of the hypersurface deformation algebra with the above modified Hamiltonian, two anomalies are found, similar to those of the vacuum case (13) and (14), but with a few additional matter terms. In order to get rid of these anomalous terms, one must demand them to be vanishing:

$$
\begin{aligned}
0= & -4\left(E^{\varphi}\right)^{2} \eta_{1} \frac{\partial\left(\eta_{2} / \eta_{1}\right)}{\partial E^{\varphi}}+8 \sqrt{E^{r}} E^{\varphi} \eta_{1} \frac{\partial\left(f / \eta_{1}\right)}{\partial E^{\varphi}} \\
& -8 \sqrt{E^{r}}\left(f-K_{r} \frac{\partial f}{\partial K_{r}}-P_{\phi} \frac{\partial f}{\partial P_{\phi}}\right) \\
& +\left[\left(E^{r}\right)^{\prime}\right]^{2} \eta_{1} \frac{\partial\left(\eta_{3} / \eta_{1}\right)}{\partial E^{\varphi}}+4\left(E^{r}\right)^{2}\left(\phi^{\prime}\right)^{2} \eta_{1} \frac{\partial\left(\eta_{4} / \eta_{1}\right)}{\partial E^{\varphi}} \\
0= & -E^{r} E^{\varphi} \eta_{1}^{2}\left(E^{\varphi}\right)^{\prime} \frac{\partial^{2}\left(f / \eta_{1}\right)}{\partial E^{\varphi} \partial K_{r}} \\
& -E^{r} E^{\varphi} \eta_{1}\left[\left(K_{r}\right)^{\prime} \frac{\partial^{2} f}{\partial K_{r}^{2}}+\left(P_{\phi}\right)^{\prime} \frac{\partial^{2} f}{\partial K_{r} \partial P_{\phi}}\right] \\
& +\left(E^{r}\right)^{\prime}\left[\left(\eta_{1}-\eta_{3}\right) \frac{E^{\varphi}}{2} \frac{\partial f}{\partial K_{r}}-E^{r} E^{\varphi} \eta_{1}^{2} \frac{\partial^{2}\left(f / \eta_{1}\right)}{\partial E^{r} \partial K_{r}}\right. \\
& \left.+\eta_{1} E^{r}\left(\frac{\partial f}{\partial K_{\varphi}}-K_{r} \frac{\partial^{2} f}{\partial K_{r} \partial K_{\varphi}}\right)\right]
\end{aligned}
$$




$$
\begin{aligned}
& +\phi^{\prime} E^{r} E^{\varphi}\left[\eta_{1}\left(\frac{P_{\phi}}{E^{\varphi}} \frac{\partial^{2} f}{\partial K_{r} \partial K_{\varphi}}-\frac{\partial^{2} f}{\partial K_{r} \partial \phi}\right)\right. \\
& \left.+2 \eta_{4} E^{r} \frac{\partial f}{\partial P_{\phi}}+\frac{\partial \eta_{1}}{\partial \phi} \frac{\partial f}{\partial K_{r}}\right] .
\end{aligned}
$$

Since none of the free functions depends on derivatives of the different variables, the coefficient of each radial derivative in the above anomalies must be vanishing. Therefore, the last two conditions can be rewritten as a set of eight differential equations. In particular, the vanishing of the coefficients of $\left[\left(E^{r}\right)^{\prime}\right]^{2}$ and $\left(\phi^{\prime}\right)^{2}$ in (38) is solved by the requirements,

$\eta_{3}\left(E^{r}, E^{\varphi}, \phi\right)=\xi_{3}\left(E^{r}\right) \eta_{1}\left(E^{r}, E^{\varphi}, \phi\right) / \xi_{1}\left(E^{r}\right)$,

$\eta_{4}\left(E^{r}, E^{\varphi}, \phi\right)=\xi_{4}\left(E^{r}\right) \eta_{1}\left(E^{r}, E^{\varphi}, \phi\right) / \xi_{1}\left(E^{r}\right)$,

where $\xi_{1}, \xi_{3}$ and $\xi_{4}$ are free functions of $E^{r}$. In addition, as in the vacuum case, we have the freedom to choose $\eta_{2}$, as it could have been absorbed inside the generic function $f$ in definition (37). For convenience, we take,

$\eta_{2}\left(E^{r}, E^{\varphi}, \phi\right)=\xi_{2}\left(E^{r}\right) \eta_{1}\left(E^{r}, E^{\varphi}, \phi\right) / \xi_{1}\left(E^{r}\right)$,

which makes the first term in Eq. (38) to vanish.

Considering now the restrictions for $f$, it is easy to see that the function

$$
\begin{aligned}
f= & \frac{\eta_{1}\left(E^{r}, E^{\varphi}, \phi\right)}{\xi_{1}\left(E^{r}\right)} \\
& \times\left[f_{0}\left(E^{r}, E^{\varphi}, \phi, K_{\varphi}, P_{\phi}\right)+K_{r} f_{1}\left(E^{r}, \phi, K_{\varphi}\right)\right],
\end{aligned}
$$

with the integration functions $f_{0}$ and $f_{1}$ provides a generic solution to the vanishing of the coefficients of $\left(K_{r}\right)^{\prime},\left(P_{\phi}\right)^{\prime}$ and $\left(E^{\varphi}\right)^{\prime}$ in (39). Thus, the dependence of the modified Hamiltonian on the radial curvature component $K_{r}$ is bound to be linear and, therefore, holonomy corrections for this variable will not be allowed. Replacing now the results (40)(43) in Eq. (38), we obtain the following condition for $f_{0}$ :

$f_{0}-P_{\phi} \frac{\partial f_{0}}{\partial P_{\phi}}-E^{\varphi} \frac{\partial f_{0}}{\partial E^{\varphi}}=0$,

which is the generalization of the vacuum relation (25). This equation restricts the form of the function $f_{0}$ in terms of the variables $E^{\varphi}$ and $P_{\phi}$. More precisely, $f_{0}$ must be of the form $f_{0}=E^{\varphi} f_{2}\left(E^{r}, \phi, K_{\varphi}, P_{\phi} / E^{\varphi}\right)$, with $f_{2}$ an integration function. Therefore, we can rewrite $f$ above, as

$$
\begin{aligned}
f= & \frac{\eta_{1}\left(E^{r}, E^{\varphi}, \phi\right)}{\xi_{1}\left(E^{r}\right)} \\
& \times\left[E^{\varphi} f_{2}\left(E^{r}, \phi, K_{\varphi}, P_{\phi} / E^{\varphi}\right)+K_{r} f_{1}\left(E^{r}, \phi, K_{\varphi}\right)\right] .
\end{aligned}
$$

Expressions (40)-(42) and (45) completely solve equation (38). Replacing all the results in the other anomaly (39), every term vanishes except for the coefficients of $\left(E^{r}\right)^{\prime}$ and $\phi^{\prime}$, which provide the following two additional restrictions:

$$
\begin{aligned}
\frac{\partial f_{2}}{\partial K_{\varphi}} & =\frac{\xi_{3}-\xi_{1}}{2 E^{r} \xi_{1}} f_{1}+\xi_{1} \frac{\partial\left(f_{1} / \xi_{1}\right)}{\partial E^{r}}, \\
\frac{\partial f_{2}}{\partial\left(P_{\phi} / E^{\varphi}\right)} & =\frac{\xi_{1}}{2 E^{r} \xi_{4}}\left(\frac{\partial f_{1}}{\partial \phi}-\frac{P_{\phi}}{E^{\varphi}} \frac{\partial f_{1}}{\partial K_{\varphi}}\right) .
\end{aligned}
$$

Note that Eq. (46) is the equivalent to (27) for this model (in order to see this, it is enough to compare the decomposition (45) with (26)). But in this case, besides (46), the functions $f_{1}$ and $f_{2}$ also need to satisfy the relation (47). This will completely fix the dependence of these functions on the angular component of the curvature $K_{\varphi}$, preventing in this way the presence of free functions of this variable. Therefore, the fundamental reason for this model not to admit holonomy corrections can be pinpointed to the existence of this last equation.

In order to solve the last two equations, let us first focus on the dependence of the functions $f_{1}$ and $f_{2}$ on the variables $K_{\varphi}$ and $P_{\phi} / E^{\varphi}$. Since the right-hand side of Eq. (46) does not contain any $P_{\phi} / E^{\varphi}$ terms, these variables cannot be coupled to $K_{\varphi}$. That is, $f_{2}$ must be a sum of two functions: $f_{2}=$ $g_{1}\left(E^{r}, \phi, P_{\phi} / E^{\varphi}\right)+g_{2}\left(E^{r}, \phi, K_{\varphi}\right)$. Plugging this form in (47) and taking the derivative of that equation with respect to $K_{\varphi}$, one obtains:

$\frac{\partial^{2} f_{1}}{\partial K_{\varphi} \partial \phi}-\frac{P_{\phi}}{E^{\varphi}} \frac{\partial^{2} f_{1}}{\partial K_{\varphi}^{2}}=0$.

It is clear that, since $f_{1}$ does not depend on $P_{\phi} / E^{\varphi}$, each term of this equation must vanish independently. Then, one concludes that $f_{1}$ is, at most, linear in $K_{\varphi}$, with the coefficient of $K_{\varphi}$ being independent of the scalar field variable $\phi$ :

$f_{1}=\xi_{1}\left(E^{r}\right)\left[f_{\phi}\left(E^{r}, \phi\right)+K_{\varphi} f_{k}\left(E^{r}\right)\right]$.

As already commented above, this result provides the explicit dependence of the function $f_{1}$ on the variables $K_{\varphi}$ and $P_{\phi} / E^{\varphi}$. Therefore, one can replace (49) in the system (46) and (47) and integrate the equations with respect to the mentioned variables. The explicit dependence of $f_{2}$ on $K_{\varphi}$ and $P_{\phi} / E^{\varphi}$ reads as follows,

$$
\begin{aligned}
f_{2}= & \xi_{1}\left(E^{r}\right)\left[f_{00}\left(E^{r}, \phi\right)+K_{\varphi} f_{10}\left(E^{r}, \phi\right)+K_{\varphi}^{2} f_{20}\left(E^{r}, \phi\right)\right. \\
& \left.+\frac{P_{\phi}}{E^{\varphi}} f_{01}\left(E^{r}, \phi\right)+\left(\frac{P_{\phi}}{E^{\varphi}}\right)^{2} f_{02}\left(E^{r}, \phi\right)\right] .
\end{aligned}
$$

Replacing the above expressions for $f_{1}(49)$ and $f_{2}(50)$ in Eqs. (46) and (47), we obtain a set of four differential relations, one for each coefficient of different powers in $K_{\varphi}$ and $P_{\phi} / E^{\varphi}$ : 
$f_{02}=-\frac{\xi_{1}}{4 E^{r} \xi_{4}} f_{k}$,

$f_{20}=\frac{\xi_{3}-\xi_{1}}{4 E^{r} \xi_{1}} f_{k}+\frac{1}{2} \frac{\partial f_{k}}{\partial E^{r}}$,

$f_{10}=\frac{\xi_{3}-\xi_{1}}{2 E^{r} \xi_{1}} f_{\phi}+\frac{\partial f_{\phi}}{\partial E^{r}}$,

$f_{01}=\frac{\xi_{1}}{2 E^{r} \xi_{4}} \frac{\partial f_{\phi}}{\partial \phi}$.

In particular, since $f_{k}$ depends only on the radial component of the triad $E^{r}$, Eqs. (51) and (52) show that neither $f_{02}$ nor $f_{20}$ depends on $\phi$. Out of the seven free functions in (49) and (50), only three will remain free after these last four equations are satisfied. The function $f_{00}$ is one of those free functions, as it does not appear in the last relations. In addition, the functions $f_{k}$ and $f_{\phi}$ will be chosen as free, whereas the remaining functions can be written in terms of these two. Finally, we rename two of the free functions with the definitions $\mathcal{V}\left(E^{r}, \phi\right):=\xi_{1} f_{00} / \sqrt{E^{r}}$ and $\xi_{0}:=-f_{k} /\left(2 \sqrt{E^{r}}\right)$ to resemble the classical form of the Hamiltonian. Taking into account all the above results, the modified Hamiltonian constraint is given by,

$$
\begin{aligned}
\widetilde{\mathcal{H}}= & \eta\left(E^{r}, E^{\varphi}, \phi\right)\left[\sqrt{E^{r}} \xi_{1}\left(\Gamma_{\varphi}^{\prime}-2 \xi_{0} K_{r} K_{\varphi}\right)\right. \\
& -\frac{E^{\varphi}}{2 \sqrt{E^{r}}}\left(\xi_{2}+\left[\xi_{0} \xi_{3}+2 E^{r} \xi_{1} \frac{\partial \xi_{0}}{\partial E^{r}}\right] K_{\varphi}^{2}-\xi_{3} \Gamma_{\varphi}^{2}\right) \\
& +\frac{\xi_{0} \xi_{1}^{2}}{\xi_{4}} \frac{P_{\phi}^{2}}{2 \sqrt{E^{r}} E^{\varphi}}+\xi_{4} \frac{\left(E^{r}\right)^{3 / 2}}{2 E^{\varphi}}\left(\phi^{\prime}\right)^{2}+\sqrt{E^{r}} E^{\varphi} \mathcal{V}\left(\phi, E^{r}\right) \\
& \left.+\xi_{1} K_{r} f_{\phi}+\frac{\xi_{1}^{2} P_{\phi}}{2 E^{r} \xi_{4}} \frac{\partial f_{\phi}}{\partial \phi}+\left(\frac{\xi_{3}-\xi_{1}}{2 E^{r}} f_{\phi}+\xi_{1} \frac{\partial f_{\phi}}{\partial E^{r}}\right) E^{\varphi} K_{\varphi}\right],
\end{aligned}
$$

where the free functions $\xi_{i}=\xi_{i}\left(E^{r}\right)$, for $i=0,1,2,3,4$, would be interpreted as inverse-triad corrections and depend only on the radial component of the triad, whereas the global factor $\eta:=\eta_{1} / \xi_{1}$ depends on all the configuration variables. In addition, the function $\mathcal{V}=\mathcal{V}\left(E^{r}, \phi\right)$ stands for a generalized potential term for the scalar field, with an arbitrary dependence on the radial triad $E^{r}$. Finally, the last free function $f_{\phi}=f_{\phi}\left(E^{r}, \phi\right)$ appears in linear terms of the momenta $K_{r}, K_{\varphi}$, and $P_{\phi}$ and couples the scalar field to curvature components. However, this function does not encode any physical information and can be removed by the following canonical transformation,

$$
\begin{aligned}
& \bar{K}_{\varphi}=K_{\varphi}-\frac{f_{\phi}}{2 \sqrt{E^{r} \xi_{0}}}, \\
& \bar{K}_{r}=K_{r}-\frac{\partial}{\partial E^{r}}\left(\frac{E^{\varphi} f_{\phi}}{2 \sqrt{E^{r}} \xi_{0}}\right), \\
& \bar{P}_{\phi}=P_{\phi}+\frac{E^{\varphi}}{2 \sqrt{E^{r}} \xi_{0}} \frac{\partial f_{\phi}}{\partial \phi} .
\end{aligned}
$$

Remarkably, this transformation leaves the diffeomorphism constraint invariant. In terms of these new variables, the final form of the modified Hamiltonian constraint reads as follows,

$$
\begin{aligned}
\widetilde{\mathcal{H}}= & \eta\left(E^{r}, E^{\varphi}, \phi\right)\left[\sqrt{E^{r}} \xi_{1}\left(\Gamma_{\varphi}{ }^{\prime}-2 \xi_{0} \bar{K}_{r} \bar{K}_{\varphi}\right)\right. \\
& -\frac{E^{\varphi}}{2 \sqrt{E^{r}}}\left(\xi_{2}+\left[\xi_{0} \xi_{3}+2 E^{r} \xi_{1} \frac{\partial \xi_{0}}{\partial E^{r}}\right] \bar{K}_{\varphi}^{2}-\xi_{3} \Gamma_{\varphi}^{2}\right) \\
& \left.+\frac{\xi_{0} \xi_{1}{ }^{2}}{\xi_{4}} \frac{\bar{P}_{\phi}^{2}}{2 \sqrt{E^{r}} E^{\varphi}}+\xi_{4} \frac{\left(E^{r}\right)^{3 / 2}}{2 E^{\varphi}}\left(\phi^{\prime}\right)^{2}+\sqrt{E^{r}} E^{\varphi} \overline{\mathcal{V}}\right],
\end{aligned}
$$

where the potential term $\mathcal{V}\left(\phi, E^{r}\right)$ has been redefined as a new generic function $\overline{\mathcal{V}}\left(\phi, E^{r}\right)$ in order to absorb the extra terms from the canonical transformation. The classical Hamiltonian is recovered when all the correction functions, $\eta$ and $\xi_{i}$, are equal to one and $\overline{\mathcal{V}}$ stands for the scalar field potential $V(\phi)$.

Notice that the dependence of this Hamiltonian on the momenta is the same as in the classical case. In particular, there are no free functions of the curvature components and thus, in contrast to the vacuum case, no holonomy corrections are allowed in this model.

Remarkably, even if we started from a generic and coupled form (37), the final modified Hamiltonian constraint, obtained through the requirement of anomaly freedom, can be expressed as a direct sum of a corrected vacuum and scalarfield Hamiltonian constraints, $\widetilde{\mathcal{H}}=\widetilde{\mathcal{H}}_{g}^{(m)}+\widetilde{\mathcal{H}}_{s}$, which read, respectively, as follows:

$$
\begin{aligned}
\widetilde{\mathcal{H}}_{g}^{(m)}:= & \eta\left[\sqrt{E^{r}} \xi_{1}\left(\Gamma_{\varphi}^{\prime}-2 \xi_{0} \bar{K}_{r} \bar{K}_{\varphi}\right)\right. \\
& \left.-\frac{E^{\varphi}}{2 \sqrt{E^{r}}}\left(\xi_{2}+\left[\xi_{0} \xi_{3}+2 E^{r} \xi_{1} \frac{\partial \xi_{0}}{\partial E^{r}}\right] \bar{K}_{\varphi}^{2}-\xi_{3} \Gamma_{\varphi}^{2}\right)\right], \\
\widetilde{\mathcal{H}}_{s}:= & \eta\left[\frac{\xi_{0} \xi_{1}^{2}}{\xi_{4}} \frac{\bar{P}_{\phi}^{2}}{2 \sqrt{E^{r}} E^{\varphi}}+\xi_{4} \frac{\left(E^{r}\right)^{3 / 2}}{2 E^{\varphi}}\left(\phi^{\prime}\right)^{2}\right. \\
& \left.+\sqrt{E^{r}} E^{\varphi} \overline{\mathcal{V}}\left(\phi, E^{r}\right)\right] .
\end{aligned}
$$

Therefore, the only non-minimal coupling between matter and geometric degrees of freedom is given by the global factor $\eta$. Note, however, that the modified Hamiltonian constraint corresponding to the geometric degrees of freedom (60) is not the same as the one obtained in the vacuum model (28). As already commented above, in this case we have less freedom than in the pure vacuum model: instead of two functions $h_{1}$ and $h_{2}$ of two variables $\left(E^{r}, K_{\varphi}\right)$ related by equation (27), in this case we have just one free function $\xi_{0}$ of the radial component of the triad $E^{r}$. 
If we now compute the Poisson brackets using the modified Hamiltonian constraint (59), the hypersurface deformation algebra takes the following form:

$$
\begin{gathered}
\left\{D\left[N^{r}\right], \widetilde{H}[N]\right\}=\widetilde{H}\left[N^{r} N^{\prime}-\left(N^{r}\right)^{\prime} N \frac{\partial \eta}{\partial E^{\varphi}} \frac{E^{\varphi}}{\eta}\right], \\
\left\{\widetilde{H}\left[N_{1}\right], \widetilde{H}\left[N_{2}\right]\right\}=D\left[\eta^{2} \xi_{0} \xi_{1}^{2} q^{r r}\left(N_{1} N_{2}^{\prime}-N_{1}^{\prime} N_{2}\right)\right] .
\end{gathered}
$$

The free functions $\eta, \xi_{0}$ and $\xi_{1}$ in the last bracket are now the ones that might produce physical effects such as signature change.

\section{Singular solutions}

Concerning the solutions not included in the previous derivation, one can obtain a modified Hamiltonian that forms an anomaly-free algebra, along with the classical diffeomorphism constraint, demanding one of the following conditions:

(i) $\eta_{1}=0, \eta_{3}=0$, and $f=f\left(E^{r}, E^{\varphi}, \phi, K_{r}, K_{\varphi}\right)$.

(ii) $\eta_{1}=0, \eta_{4}=0$, and $f=f\left(E^{r}, E^{\varphi}, \phi, K_{\varphi}, P_{\phi}\right)$.

(iii) $\eta_{1}=0$, and $f=f\left(E^{r}, E^{\varphi}, \phi, K_{\varphi}\right)$.

(iv) $\eta_{1}=0, \eta_{3}=0$, and $\eta_{4}=0$.

Taking $\eta_{1}=0$, one removes the radial derivative of the spinconnection and, thus, terms containing $\left(E^{r}\right)^{\prime \prime}$ and $\left(E^{\varphi}\right)^{\prime}$ are pulled out from the Hamiltonian. In addition, two further conditions are required. For example, one can demand $\eta_{3}=$ 0 , removing all radial derivatives of the triad components and that the Hamiltonian does not depend on the scalar field momentum, that is, case (i). Alternatively, one can take $\eta_{4}=$ 0 , which would remove the radial derivatives of the scalar field, and request that the Hamiltonian does not depend on $K_{r}$ (case (ii)). Another possibility, (iii), implies the removal of the momenta $K_{r}$ and $P_{\phi}$ from the Hamiltonian, leaving $\eta_{3}$ and $\eta_{4}$ free. The last option would be to eliminate all radial derivatives (case (iv)). As in the pure vacuum model, this last case resembles the corresponding homogeneous spacetime.

In summary, holonomy corrections for a given momentum $\left(K_{r}, K_{\varphi}, P_{\phi}\right)$ are incompatible with the presence of the radial derivative of its corresponding conjugate variable $\left(\left(E^{r}\right)^{\prime},\left(E^{\varphi}\right)^{\prime}, \phi^{\prime}\right)$ in the modified Hamiltonian. Nevertheless, taking into account the BKL conjecture, one could consider a model where, as small values of the triad components were approached, certain correction functions would be vanishing from a given value on, annihilating in this way radial derivatives in the Hamiltonian and entering the homogeneous BKL limit. In this homogeneous region, holonomy corrections would be allowed with no further restriction arising from anomaly freedom. Such a model would be described, for instance, by a modified Hamiltonian of the form,

$$
\begin{aligned}
\tilde{\mathcal{H}}= & \frac{\tilde{f}_{1}\left(P_{\phi}\right)}{2 \sqrt{E^{r}} E^{\varphi}}-2 \sqrt{E^{r}} \tilde{f}_{2}\left(K_{r}, K_{\varphi}\right)-\frac{E^{\varphi}}{2 \sqrt{E^{r}}} \tilde{f}_{3}\left(K_{\varphi}\right) \\
& +\eta_{1} \sqrt{E^{r}} \Gamma_{\varphi}^{\prime}+\eta_{3} \frac{E^{\varphi} \Gamma_{\varphi}^{2}}{2 \sqrt{E^{r}}}+\eta_{4} \frac{\left(E^{r}\right)^{3 / 2}}{2 E^{\varphi}}\left(\phi^{\prime}\right)^{2} .
\end{aligned}
$$

This Hamiltonian would form a first-class algebra with the classical diffeomorphism constraint if $\widetilde{f}_{1}, \widetilde{f}_{2}$, and $\widetilde{f}_{3}$ took their corresponding classical value as long as $\eta_{1}, \eta_{3}$, and $\eta_{4}$ were non-vanishing. In the region of the configuration space where $\eta_{1}=\eta_{3}=\eta_{4}=0$, the new functions $\widetilde{f}_{1}, \widetilde{f}_{1}$, and $\widetilde{f_{3}}$ would be completely free and could encode, for instance, holonomy modifications.

\subsection{Dust}

In spherical symmetry, a dust field can be described in terms of a scalar variable $\Phi$ and its conjugate momentum $P_{\Phi}$, with Poisson brackets $\left\{\Phi(x), P_{\Phi}(y)\right\}=\delta(x-y)$. The classical contributions of the dust to the Hamiltonian and diffeomorphism constraints are respectively given by,

$$
\begin{aligned}
& \mathcal{H}_{\Phi}=P_{\Phi} \sqrt{1+\frac{E^{r}\left(\Phi^{\prime}\right)^{2}}{\left(E^{\varphi}\right)^{2}}}, \\
& \mathcal{D}_{\Phi}=\Phi^{\prime} P_{\Phi} .
\end{aligned}
$$

Even if, due to the presence of the square root in the dust Hamiltonian constraint, the computations will be a bit more complicated than in the previous case, the procedure will be similar. As the starting point of our analysis, we will consider the following general form for the modified Hamiltonian that describes the dynamics of the dust model coupled to gravity,

$$
\begin{aligned}
\widetilde{\mathcal{H}}= & f\left(E^{r}, E^{\varphi}, \Phi, K_{r}, K_{\varphi}, P_{\Phi}\right)+\eta_{1}\left(E^{r}, E^{\varphi}, \Phi\right) \sqrt{E^{r}} \Gamma_{\varphi}^{\prime} \\
& -\eta_{2}\left(E^{r}, E^{\varphi}, \Phi\right) \frac{E^{\varphi}}{2 \sqrt{E^{r}}}+\eta_{3}\left(E^{r}, E^{\varphi}, \Phi\right) \frac{E^{\varphi} \Gamma_{\varphi}^{2}}{2 \sqrt{E^{r}}} \\
& +g\left(E^{r}, E^{\varphi}, \Phi, K_{r}, K_{\varphi}, P_{\Phi}\right) \sqrt{1+\eta_{4}\left(E^{r}, E^{\varphi}, \Phi\right) \frac{E^{r}\left(\Phi^{\prime}\right)^{2}}{\left(E^{\varphi}\right)^{2}}} .
\end{aligned}
$$

Let us define the shorthand notation

$\mathcal{S}:=\sqrt{1+\eta_{4}\left(E^{r}, E^{\varphi}, \Phi\right) \frac{E^{r}\left(\Phi^{\prime}\right)^{2}}{\left(E^{\varphi}\right)^{2}}}$,

to encode the square root.

Notice that we have introduced two generic functions, $f$ and $g$, that might depend on all the variables of the model, but not on their radial derivatives. These functions replace the classical terms that go with the momenta $K_{r}, K_{\varphi}$, and $P_{\Phi}$. More precisely, the function $f$ replaces the quadratic combination of curvature components in the geometric Hamiltonian, whereas $g$ takes the place of the dust field momen- 
tum $P_{\Phi}$. In this way, these two functions might describe the polymerization of the momenta but also, as they depend on the configuration variables, might stand for inverse-triad corrections. In addition, their generic form is suitable for any kind of coupling between geometric and matter degrees of freedom. Finally, the functions $\eta_{i}$, with $i=1,2,3,4$, are allowed to depend on the triad components $E^{r}$ and $E^{\varphi}$ in order to stand for possible inverse-triad corrections. Additionally, they might also depend on the dust field, enabling a generic coupling.

As in the previous models, two anomalies are obtained when the Poisson algebra is computed. In order to obtain a first class algebra, we demand that both anomalous terms vanish:

$$
\begin{aligned}
& 0=2 \eta_{1}\left(E^{\varphi}\right)^{2} \mathcal{S} \frac{\partial\left(\eta_{2} / \eta_{1}\right)}{\partial E^{\varphi}}-4 \eta_{1} \sqrt{E^{r}} E^{\varphi} \mathcal{S} \frac{\partial}{\partial E^{\varphi}}\left[\frac{f+g \mathcal{S}}{\eta_{1}}\right] \\
& +g \eta_{1} \eta_{4}\left(\Phi^{\prime}\right)^{2} \frac{\left(E^{r}\right)^{3 / 2}}{\left(E^{\varphi}\right)^{2}} \\
& +4 \sqrt{E^{r}} \mathcal{S}\left(1-P_{\Phi} \frac{\partial}{\partial P_{\Phi}}-K_{r} \frac{\partial}{K_{r}}\right)(f+\mathcal{S} g) \\
& -\eta_{1}\left[\left(E^{r}\right)^{\prime}\right]^{2} \mathcal{S} \frac{\partial\left(\eta_{3} / \eta_{1}\right)}{\partial E^{\varphi}} \\
& 0=\eta_{1} \eta_{4} E^{r}\left(E^{\varphi}\right)^{-1} \Phi^{\prime} \Phi^{\prime \prime} \frac{\partial g}{\partial K_{r}} \\
& +\eta_{1} E^{\varphi} \mathcal{S}\left(\left(K_{r}\right)^{\prime} \frac{\partial}{\partial K_{r}}+\left(P_{\Phi}\right)^{\prime} \frac{\partial}{\partial P_{\Phi}}\right)\left(\frac{\partial f}{\partial K_{r}}+\mathcal{S} \frac{\partial g}{\partial K_{r}}\right) \\
& -\eta_{1}\left(E^{r}\right)^{\prime} \mathcal{S}\left[\left(1-K_{r} \frac{\partial}{\partial K_{r}}\right)\left(\frac{\partial f}{\partial K_{\varphi}}+\mathcal{S} \frac{\partial g}{\partial K_{\varphi}}\right)\right. \\
& \left.+E^{\varphi}\left(\frac{\eta_{1}-\eta_{3}}{2 E^{r}}-\frac{\partial}{\partial E^{r}}\right)\left(\frac{\partial\left(f / \eta_{1}\right)}{\partial K_{r}}+\mathcal{S} \frac{\partial\left(g / \eta_{1}\right)}{\partial K_{r}}\right)\right] \\
& -\Phi^{\prime} \mathcal{S}\left[\eta_{1}\left(P_{\Phi} \frac{\partial}{\partial K_{\varphi}}-E^{\varphi} \frac{\partial}{\partial \Phi}\right)\left(\frac{\partial f}{\partial K_{r}}+\mathcal{S} \frac{\partial g}{\partial K_{r}}\right)\right. \\
& +E^{\varphi} \frac{\partial \eta_{1}}{\partial \Phi}\left(\frac{\partial f}{\partial K_{r}}+\mathcal{S} \frac{\partial g}{\partial K_{r}}\right) \\
& \left.+2 \sqrt{E^{r}} \eta_{4} g\left(\mathcal{S}^{-1} \frac{\partial f}{\partial P_{\Phi}}+\frac{\partial g}{\partial P_{\Phi}}\right)\right] \\
& +\eta_{1}^{2} E^{\varphi}\left(E^{\varphi}\right)^{\prime} \mathcal{S}\left[\frac{\partial}{\partial E^{\varphi}}\left(\frac{\partial\left(f / \eta_{1}\right)}{\partial K_{r}}+\mathcal{S} \frac{\partial\left(g / \eta_{1}\right)}{\partial K_{r}}\right)\right] .
\end{aligned}
$$

Due to the presence of the square root in $\mathcal{S}$ and the coupling between $P_{\Phi}$ and $\Phi^{\prime}$, these anomalies are a bit more involved than those in Sect. 4.1. Nevertheless, some conditions for the free functions can immediately be read out. In particular, from the vanishing of the coefficient of $\left[\left(E^{r}\right)^{\prime}\right]^{2}$ in equation (69), one obtains:

$\eta_{3}\left(E^{r}, E^{\varphi}, \Phi\right)=\xi_{3}\left(E^{r}\right) \eta_{1}\left(E^{r}, E^{\varphi}, \Phi\right) / \xi_{1}\left(E^{r}\right)$.
In addition, as in previous cases, we use the freedom in the definition of the function $\eta_{2}$ to choose,

$\eta_{2}\left(E^{r}, E^{\varphi}, \Phi\right)=\xi_{2}\left(E^{r}\right) \eta_{1}\left(E^{r}, E^{\varphi}, \Phi\right) / \xi_{1}\left(E^{r}\right)$,

which annihilates the first term of the anomaly (69). The first term in (70) is the only one with second-order radial derivatives of the dust field and must vanish on its own. This demand is satisfied when the function $g$ does not depend on $K_{r}$. Therefore, we will write this function as,

$g=\eta_{1}\left(E^{r}, E^{\varphi}, \phi\right) g_{0}\left(E^{r}, E^{\varphi}, K_{\varphi}, \Phi, P_{\Phi}\right) / \xi_{1}\left(E^{r}\right)$,

where the function $\eta_{1} / \xi_{1}$ has been introduced as a global factor for convenience. As there is no more dependence on radial derivatives of the variables, the coefficients of $\left(K_{r}\right)^{\prime},\left(P_{\Phi}\right)^{\prime}$, and $\left(E^{\varphi}\right)^{\prime}$ in the anomaly (70) must also vanish by themselves. Taking into account (73), three independent equations for the function $f$ are obtained:

$$
\begin{aligned}
& 0=\frac{\partial^{2} f}{\partial K_{r}^{2}}, \\
& 0=\frac{\partial^{2} f}{\partial K_{r} \partial P_{\Phi}}, \\
& 0=\frac{\partial^{2}\left(f / \eta_{1}\right)}{\partial E^{\varphi} \partial K_{r}} .
\end{aligned}
$$

It is straightforward to see that the general solution for this system can be written in terms of two free functions, $f_{0}$ and $f_{1}$, as follows,

$$
f=\frac{\eta_{1}}{\xi_{1}}\left[f_{0}\left(E^{r}, E^{\varphi}, \Phi, K_{\varphi}, P_{\Phi}\right)+K_{r} f_{1}\left(E^{r}, \Phi, K_{\varphi}\right)\right] .
$$

At this point, we already see that the modified Hamiltonian will be linear in $K_{r}$ and that this variable will be decoupled from the momentum of the dust field $P_{\Phi}$.

The replacement of (71)-(73) and (77) in the anomaly equations (69) and (70) leads to a much simpler version of these two equations:

$$
\begin{aligned}
0= & \mathcal{S}\left(f_{0}-E^{\varphi} \frac{\partial f_{0}}{\partial E^{\varphi}}-P_{\Phi} \frac{\partial f_{0}}{\partial P_{\Phi}}\right) \\
& +\left(g_{0}-E^{\varphi} \frac{\partial g_{0}}{\partial E^{\varphi}}-P_{\Phi} \frac{\partial g_{0}}{\partial P_{\Phi}}\right) \\
& +\frac{\eta_{4} E^{r}}{\left(E^{\varphi}\right)^{2}}\left(\Phi^{\prime}\right)^{2}\left(-\frac{E^{\varphi}}{2} \frac{g_{0}}{\eta_{4}} \frac{\partial \eta_{4}}{\partial E^{\varphi}}\right. \\
& \left.+g_{0}-E^{\varphi} \frac{\partial g_{0}}{\partial E^{\varphi}}-P_{\Phi} \frac{\partial g_{0}}{\partial P_{\Phi}}\right), \\
0= & \Phi^{\prime}\left[P_{\Phi} \frac{\partial f_{1}}{\partial K_{\varphi}}-E^{\varphi} \frac{\partial f_{1}}{\partial \Phi}\right. \\
& \left.+2 \sqrt{E^{r}} \eta_{4} \frac{g_{0}}{\xi_{1}}\left(\mathcal{S}^{-1} \frac{\partial f_{0}}{\partial P_{\Phi}}+\frac{\partial g_{0}}{\partial P_{\Phi}}\right)\right]
\end{aligned}
$$




$$
\begin{aligned}
& +\left(E^{r}\right)^{\prime}\left[E^{\varphi}\left(\frac{\xi_{1}-\xi_{3}}{2 E^{r} \xi_{1}} f_{1}-\xi_{1} \frac{\partial\left(f_{1} / \xi_{1}\right)}{\partial E^{r}}\right)\right. \\
& \left.+\frac{\partial f_{0}}{\partial K_{\varphi}}+\mathcal{S} \frac{\partial g_{0}}{\partial K_{\varphi}}\right]
\end{aligned}
$$

In fact, looking at the dependence on the radial derivatives of the variables, these two equations can be rewritten as a set of seven independent equations. From (78), one gets the following three differential equations,

$$
\begin{aligned}
0= & g_{0}-E^{\varphi} \frac{\partial g_{0}}{\partial E^{\varphi}}-P_{\Phi} \frac{\partial g_{0}}{\partial P_{\Phi}}, \\
0= & f_{0}-E^{\varphi} \frac{\partial f_{0}}{\partial E^{\varphi}}-P_{\Phi} \frac{\partial f_{0}}{\partial P_{\Phi}}, \\
0= & \frac{E^{\varphi}}{2} \frac{g_{0}}{\eta_{4}} \frac{\partial \eta_{4}}{\partial E^{\varphi}} \\
& -\left(g_{0}-E^{\varphi} \frac{\partial g_{0}}{\partial E^{\varphi}}-P_{\Phi} \frac{\partial g_{0}}{\partial P_{\Phi}}\right),
\end{aligned}
$$

whereas (79) provides four additional conditions:

$$
\begin{aligned}
& 0=\frac{\partial f_{0}}{\partial P_{\Phi}}, \\
& 0=\frac{\partial g_{0}}{\partial K_{\varphi}}, \\
& 0=P_{\Phi} \frac{\partial f_{1}}{\partial K_{\varphi}}-E^{\varphi} \frac{\partial f_{1}}{\partial \Phi}+2 \sqrt{E^{r}} \eta_{4} \frac{g_{0}}{\xi_{1}} \frac{\partial g_{0}}{\partial P_{\Phi}}, \\
& 0=E^{\varphi}\left(\frac{\xi_{1}-\xi_{3}}{2 E^{r} \xi_{1}} f_{1}-\xi_{1} \frac{\partial\left(f_{1} / \xi_{1}\right)}{\partial E^{r}}\right)+\frac{\partial f_{0}}{\partial K_{\varphi}} .
\end{aligned}
$$

Equation (83) demands $f_{0}$ to be independent of $P_{\Phi}$ and one deduces that $g_{0}$ cannot depend on $K_{\varphi}$ from Eq. (84). Hence, there will not be holonomy modifications multiplying the $\Phi^{\prime}$ term of the Hamiltonian. Using these results, the general solutions to (80) and (81) are respectively given by:

$$
\begin{aligned}
& g_{0}=E^{\varphi} \widetilde{g_{0}}\left(E^{r}, \Phi, \frac{P_{\Phi}}{E^{\varphi}}\right), \\
& f_{0}=E^{\varphi} \widetilde{f_{0}}\left(E^{r}, K_{\varphi}, \Phi\right) .
\end{aligned}
$$

Note that the term between brackets in Eq. (82) is equal to the right-hand side of Eq. (80), so it must vanish. Therefore, it is straightforward to integrate (82) to obtain that $\eta_{4}=$ $1 / \xi_{4}\left(E^{r}, \Phi\right)$, with the free integration function $\xi_{4}$.

From the system (80)-(86), only two differential equations are left to be solved, namely (85) and (86). Enforcing the solutions for the other equations, relations (85) and (86) are reduced to the following form:

$$
\frac{\partial \tilde{f}_{0}}{\partial K_{\varphi}}=\frac{\xi_{3}-\xi_{1}}{2 E^{r} \xi_{1}} f_{1}+\xi_{1} \frac{\partial\left(f_{1} / \xi_{1}\right)}{\partial E^{r}}
$$

$\frac{\partial\left({\widetilde{g_{0}}}^{2}\right)}{\partial\left(P_{\Phi} / E^{\varphi}\right)}=\frac{\xi_{1} \xi_{4}}{\sqrt{E^{r}}}\left(\frac{\partial f_{1}}{\partial \Phi}-\frac{P_{\Phi}}{E^{\varphi}} \frac{\partial f_{1}}{\partial K_{\varphi}}\right)$.

If we differentiate the first one with respect to $K_{\varphi}$, two independent conditions arise:

$$
\begin{aligned}
& 0=\frac{\partial^{2} f_{1}}{\partial K_{\varphi}^{2}}, \\
& 0=\frac{\partial^{2} f_{1}}{\partial K_{\varphi} \partial \Phi},
\end{aligned}
$$

which are solved by the expression,

$f_{1}=\xi_{1}\left(E^{r}\right)\left[f_{\Phi}\left(E^{r}, \Phi\right)-2 \sqrt{E^{r}} \xi_{0}\left(E^{r}\right) K_{\varphi}\right]$.

The integration functions $\xi_{0}$ and $f_{\Phi}$ have been chosen to resemble the notation of previous sections. Plugging this form for $f_{1}$ in (89) and (90), it is straightforward to integrate those two equations and obtain the general form of the functions $\widetilde{g}_{0}$ and $\widetilde{f}_{0}$ :

$$
\begin{aligned}
\tilde{g}_{0}= & \xi_{1} \sqrt{\xi_{4}} \sqrt{\xi_{0}\left(\frac{P_{\Phi}}{E^{\varphi}}\right)^{2}+\frac{P_{\Phi}}{\sqrt{E^{r}} E^{\varphi}} \frac{\partial f_{\Phi}}{\partial \Phi}+\mathcal{V}_{1}} \\
\tilde{f}_{0}= & -\left(\frac{\xi_{0} \xi_{3}}{2 \sqrt{E^{r}}}+\sqrt{E^{r}} \xi_{1} \frac{\partial \xi_{0}}{\partial E^{r}}\right) K_{\varphi}^{2} \\
& +\left(\frac{\xi_{3}-\xi_{1}}{2 E^{r}} f_{\Phi}+\xi_{1} \frac{\partial f_{\Phi}}{\partial E^{r}}\right) K_{\varphi}+\mathcal{V}_{2}
\end{aligned}
$$

The terms $\mathcal{V}_{1}\left(E^{r}, \Phi\right)$ and $\mathcal{V}_{2}\left(E^{r}, \Phi\right)$ are completely free integration functions.

Finally, collecting all the results, one arrives to the following modified Hamiltonian constraint:

$$
\begin{aligned}
\widetilde{\mathcal{H}}= & \eta\left(E^{r}, E^{\varphi}, \Phi\right)\left[\sqrt{E^{r}} \xi_{1}\left(\Gamma_{\varphi}^{\prime}-2 \xi_{0} K_{r} K_{\varphi}\right)\right. \\
& -\frac{E^{\varphi}}{2 \sqrt{E^{r}}}\left(\xi_{2}+\left[\xi_{0} \xi_{3}+2 E^{r} \xi_{1} \frac{\partial \xi_{0}}{\partial E^{r}}\right] K_{\varphi}^{2}-\xi_{3} \Gamma_{\varphi}^{2}\right) \\
& +\xi_{1} \sqrt{\xi_{0} P_{\Phi}^{2}+\frac{E^{\varphi} P_{\Phi}}{\sqrt{E^{r}}} \frac{\partial f_{\Phi}}{\partial \Phi}+\left(E^{\varphi}\right)^{2} \mathcal{V}_{1}} \sqrt{\xi_{4}+\frac{E^{r}\left(\Phi^{\prime}\right)^{2}}{\left(E^{\varphi}\right)^{2}}} \\
& +\sqrt{E^{r}} E^{\varphi} \mathcal{V}_{2} \\
& \left.+\xi_{1} K_{r} f_{\Phi}+\left(\frac{\xi_{3}-\xi_{1}}{2 E^{r}} f_{\Phi}+\xi_{1} \frac{\partial f_{\Phi}}{\partial E^{r}}\right) E^{\varphi} K_{\varphi}\right]
\end{aligned}
$$

with the global factor defined as $\eta:=\eta_{1} / \xi_{1}$. Note that $f_{\Phi}$ plays a similar role as the function $f_{\phi}$ introduced in the previous section, and appears multiplying linear terms of the momenta. Indeed, as happened with $f_{\phi}$, one can perform a canonical transformation that removes it from the Hamiltonian and preserves the form of the diffeomorphism constraint: 
$\bar{K}_{\varphi}=K_{\varphi}-\frac{f_{\Phi}}{2 \xi_{0} \sqrt{E^{r}}}$,

$\bar{K}_{r}=K_{r}-\frac{\partial}{\partial E^{r}}\left(\frac{E^{\varphi} f_{\Phi}}{2 \sqrt{E^{r}} \xi_{0}}\right)$,

$\bar{P}_{\Phi}=P_{\Phi}+\frac{E^{\varphi}}{2 \xi_{0} \sqrt{E^{r}}} \frac{\partial f_{\Phi}}{\partial \Phi}$.

Considering these new canonical variables, the modified Hamiltonian constraint reads:

$$
\begin{aligned}
\widetilde{\mathcal{H}}= & \eta\left(E^{r}, E^{\varphi}, \Phi\right)\left[\sqrt{E^{r}} \xi_{1}\left(\Gamma_{\varphi}^{\prime}-2 \xi_{0} \bar{K}_{r} \bar{K}_{\varphi}\right)\right. \\
& -\frac{E^{\varphi}}{2 \sqrt{E^{r}}}\left(\xi_{2}+\left[\xi_{0} \xi_{3}+2 E^{r} \xi_{1} \frac{\partial \xi_{0}}{\partial E^{r}}\right] \bar{K}_{\varphi}^{2}-\xi_{3} \Gamma_{\varphi}^{2}\right) \\
& \left.+\xi_{1} \sqrt{\xi_{0} \bar{P}_{\Phi}^{2}+\left(E^{\varphi}\right)^{2} \overline{\mathcal{V}}_{1}} \sqrt{\xi_{4}+\frac{E^{r}\left(\Phi^{\prime}\right)^{2}}{\left(E^{\varphi}\right)^{2}}}+\sqrt{E^{r}} E^{\varphi} \overline{\mathcal{V}}_{2}\right] .
\end{aligned}
$$

The deformation obtained for the dust matter content is quite similar to the one presented for the scalar field. The five free functions of the radial component of the triad $\xi_{i}\left(E^{r}\right)$, with $i=0,1,2,3$, as well as $\xi_{4}=\xi_{4}\left(E^{r}, \Phi\right)$, encode possible inverse-triad corrections. The formalism also admits two free functions $\overline{\mathcal{V}}_{1}\left(E^{r}, \Phi\right)$ and $\overline{\mathcal{V}}_{2}\left(E^{r}, \Phi\right)$ that depend on the dust field and on the radial component of the triad. The former one modifies the kinetic energy of the dust field, whereas the latter can be interpreted as a potential term. In fact, in the particular case this potential acquired a constant value, it would reproduce the cosmological constant. The classical form of the Hamiltonian (with a vanishing cosmological constant) is obtained for the case $\xi_{i}=1$, with $i=0,1,2,3$, 4, and $\overline{\mathcal{V}}_{1}=\overline{\mathcal{V}}_{2}=0$.

Note that the algebra obtained for this modified Hamiltonian and the one derived for the scalar-field case are equal and it is given by relations (4a), (62), and (63). Furthermore, the modified Hamiltonian constraint (100) can also be separated into geometric and matter parts, $\widetilde{\mathcal{H}}=\widetilde{\mathcal{H}}_{g}^{(m)}+\widetilde{\mathcal{H}}_{d}$, with $\widetilde{\mathcal{H}}_{g}^{(m)}$ being equal to the modified geometric Hamiltonian (60) obtained for the scalar field model. The dust contribution is given by,

$\widetilde{\mathcal{H}}_{d}:=\eta\left[\xi_{1} \sqrt{\xi_{0} \bar{P}_{\Phi}^{2}+\left(E^{\varphi}\right)^{2} \overline{\mathcal{V}}_{1}} \sqrt{\xi_{4}+\frac{E^{r}\left(\Phi^{\prime}\right)^{2}}{\left(E^{\varphi}\right)^{2}}}+\sqrt{E^{r}} E^{\varphi} \overline{\mathcal{V}}_{2}\right]$.

Finally, a brief comment about solutions not contained in the previous derivation, which are also quite similar to the ones presented for the scalar-field model. More specifically, we find the following four different families of singular solutions:

(i) $\eta_{1}=0, \eta_{3}=0$ and $f, g$ do not depend on $P_{\Phi}$. (ii) $\eta_{1}=0, \eta_{4}=0$ and $f, g$ do not depend on $K_{r}$.

(iii) $\eta_{1}=0$ and $f, g$ do not depend on $K_{r}$ nor $P_{\Phi}$.

(iv) $\eta_{1}=0, \eta_{3}=0$ and $\eta_{4}=0$.

Each of these conditions provides a consistent modification of the classical Hamiltonian constraint when replaced in expression (67). Once again, the last option corresponds to the homogeneous limit.

\section{Conclusions}

In this paper, we have considered possible inverse-triad and holonomy modifications for spherically symmetric models in vacuum as well as coupled to simple matter models. For such a purpose, the generic forms (12), (37) and (67) of the modified Hamiltonian constraint have been proposed, for vacuum, scalar-field and dust model, respectively. In these expressions we have left complete freedom for the dependence on the triad and curvature components; whereas the radial derivatives of the variables have been left as in the classical Hamiltonian. In particular, we have allowed for non-minimal couplings between geometric and matter degrees of freedom. Even if the classical model is minimally coupled to matter, it might happen that, as we approach the quantum regime, non-minimal couplings are developed. In addition, possible polymerization of matter fields are also considered in the mentioned modified Hamiltonians.

The modification of the diffeomorphism constraint has only been considered for the vacuum model. Firstly, the full theory of loop quantum gravity does not regularize this constraint and, therefore, such corrections are not expected. Secondly, in Appendix A we consider a quite generic deformation of the diffeomorphism constraint for the vacuum model and show that, under the requirement of forming a closed algebra, the only allowed modification is a global multiplicative function, in agreement with results presented in [38]. This points out that the deformation of the diffeomorphism constraint is trivial and does not provide additional freedom for further corrections in the Hamiltonian.

The requirement that these modified constraints form a first-class algebra turns out to be quite restrictive. For each model (vacuum, scalar field and dust), the final expression for the modified Hamiltonian ((28), (59) and (100), respectively) has been obtained. These modified Hamiltonians are the main result of our study and represent a family of consistent deformations of spherically symmetric general relativity.

Note that, apart from the (trivial) global multiplicative function, none of these Hamiltonians contain free functions of the angular component of the triad $E^{\varphi}$, which rules out the possibility of including inverse-triad corrections associated to this component. In addition, only the vacuum Hamiltonian (28) presents free functions ( $h_{1}$ and $h_{2}$ ) of the angular cur- 
vature component $K_{\varphi}$. Both are related through (27) and can be interpreted as holonomy corrections. On the other hand, no holonomy modifications involving the radial component of the curvature $K_{r}$ are allowed, not even in vacuum. Furthermore, the dependence of the modified Hamiltonian on the momenta of the matter sector is also bound to its classical form. Hence, no polymerization of the matter variables is allowed under the present assumptions.

In our analysis we have shown that the absence of corrections involving $E^{\varphi}$ and $K_{r}$ can be understood from the requirement of mathematical consistency of the effective theory. Nonetheless, inverse-triad corrections for $E^{\varphi}$ and holonomy modifications including $K_{r}$ are not usually considered in the literature with some justification on physical grounds. First, in spherical symmetry, the area of the underlying elementary plaquettes naturally depends on $E^{r}$ but not on $E^{\varphi}$ and, thus, inverse-triad corrections are expected to depend only on the radial component of the triad. Second, for the non-compact radial direction, one would need to consider non-local holonomy corrections for $K_{r}$. Although a proposal in this direction (based on a truncated expansion on derivatives of $K_{r}$ ) can be found in reference [51], the implementation of such non-local modifications remains as an open question.

Contrary to the vacuum case, no holonomy corrections are allowed when matter fields are included. This is a direct consequence of the presence of the $\phi^{\prime}$ and $\Phi^{\prime}$ terms in the anomaly equations (39) and (70), respectively. Whereas in the vacuum case the two remaining free functions ( $h_{1}$ and $h_{2}$ ) are required to fulfill a unique relation, (27), in each matter model an additional restriction arises, (47) and (90). This further condition completely fixes the dependence of the Hamiltonian on the angular curvature component $K_{\varphi}$ to its classical form and leaves no room for holonomy corrections.

In addition, we have also found some singular solutions (in the sense that they are not contained in the general solutions leading to the forms (28), (59) and (100) of the Hamiltonian) to the anomaly equations that provide an anomaly-free algebra. These solutions provide a consistent deformation of the classical Hamiltonian assuming the vanishing of one (or several) correction functions. In these particular scenarios, different radial derivatives of the configuration variables are removed and a higher degree of freedom is acquired for the remaining terms. In particular, holonomy corrections are allowed in each case (see, for instance, the example (64) for a scalar-field matter content). We conclude that the presence of radial derivatives of a given variable in the Hamiltonian is the main restriction regarding the polymerization of its canonical counterpart.

Finally, let us comment that the main limitation of our analysis is that no free dependence on variables with radial derivatives has been allowed in the generic form of the modified Hamiltonians. Nonetheless, our results point out quite generically to the impossibility of including holonomy corrections in the presence of matter fields making use of the real-valued Ashtekar-Barbero variables. As an alternative, in the last few years, some proposals have been made by considering self-dual variables [47-50].

Acknowledgements $\mathrm{AAB}$ acknowledges financial support from the FPI PRE2018-086516 fellowship of the Spanish Ministry of Science, Innovation and Universities. This work is funded by Project FIS201785076-P (MINECO/AEI/FEDER, UE) and Basque Government Grant no. IT956-16.

Data Availability Statement This manuscript has no associated data or the data will not be deposited. [Authors' comment: This is a theoretical article with no additional data.]

Open Access This article is licensed under a Creative Commons Attribution 4.0 International License, which permits use, sharing, adaptation, distribution and reproduction in any medium or format, as long as you give appropriate credit to the original author(s) and the source, provide a link to the Creative Commons licence, and indicate if changes were made. The images or other third party material in this article are included in the article's Creative Commons licence, unless indicated otherwise in a credit line to the material. If material is not included in the article's Creative Commons licence and your intended use is not permitted by statutory regulation or exceeds the permitted use, you will need to obtain permission directly from the copyright holder. To view a copy of this licence, visit http://creativecomm ons.org/licenses/by/4.0/.

Funded by $\mathrm{SCOAP}^{3}$.

\section{Appendix A: Modified diffeomorphism constraint in vac- uum}

In this appendix, we will consider a deformed form for the diffeomorphism constraint in vacuum and demand that it closes algebra along with the deformed Hamiltonian constraint (12). The result will be that the only allowed correction for the diffeomorphism constraint is a global function, which generalizes the previous result presented in [38].

We will allow for a multiplicative correction function on each term of the diffeomorphism constraint or, equivalently, a global function $\Omega$ and a correction function $\omega$ on one of the terms. In this way, we start our analysis from the general form,

$$
\begin{aligned}
& \widetilde{\mathcal{D}}_{g}=\Omega\left(E^{r}, E^{\varphi}, K_{r}, K_{\varphi}\right) \\
& \quad \times\left[\left(K_{\varphi}\right)^{\prime} E^{\varphi}-\omega\left(E^{r}, E^{\varphi}, K_{r}, K_{\varphi}\right) K_{r}\left(E^{r}\right)^{\prime}\right] .
\end{aligned}
$$

with both corrections depending on all the four variables.

This diffeomorphism constraint along with the modified Hamiltonian (12) produces five different anomalous terms:

$$
\begin{aligned}
\left\{\widetilde{D}_{g}\left[N_{1}^{r}\right], \widetilde{D}_{g}\left[N_{2}^{r}\right]\right\} & \rightarrow \mathcal{A}_{\mathcal{D}}^{D D}, \\
\left\{\widetilde{D}_{g}\left[N^{r}\right], \widetilde{H}_{g}[N]\right\} & \rightarrow \mathcal{A}_{\mathcal{D}}^{D H 0}, \mathcal{A}_{\mathcal{D}}^{D H 1}, \mathcal{A}_{\mathcal{D}}^{D H 2}, \\
\left\{\widetilde{H}_{g}\left[N_{1}\right], \widetilde{H}_{g}\left[N_{2}\right]\right\} & \rightarrow \mathcal{A}_{\mathcal{D}}^{H H} .
\end{aligned}
$$


The anomalous terms $\mathcal{A}_{\mathcal{D}}^{D H 0}, \mathcal{A}_{\mathcal{D}}^{D H 1}$, and $\mathcal{A}_{\mathcal{D}}^{D H 2}$ from the second bracket are multiplied by $N^{r},\left(N^{r}\right)^{\prime}$ and $\left(N^{r}\right)^{\prime \prime}$, respectively, and thus they must vanish independently. From now on, we will exclude irrelevant global factors.

Let us first focus on $\mathcal{A}_{\mathcal{D}}^{H H}$ :

$$
\begin{aligned}
\mathcal{A}_{\mathcal{D}}^{H H}:= & \left(E^{r}\right)^{\prime}\left[\frac{E^{\varphi}}{2 E^{r}}\left(\eta_{1}-\eta_{3}+2 E^{r} \frac{\partial \eta_{1}}{\partial E^{r}}\right) \frac{\partial f_{g}}{\partial K_{r}}\right. \\
& \left.+\eta_{1}\left(\frac{\partial f_{g}}{\partial K_{\varphi}}-\omega K_{r} \frac{\partial^{2} f_{g}}{\partial K_{\varphi} \partial K_{r}}-E^{\varphi} \frac{\partial^{2} f_{g}}{\partial E^{r} \partial K_{r}}\right)\right] \\
& +E^{\varphi}\left(E^{\varphi}\right)^{\prime}\left[\eta_{1} \frac{\partial^{2} f_{g}}{\partial E^{\varphi} \partial K_{r}}-\frac{\partial \eta_{1}}{\partial E^{\varphi}} \frac{\partial f_{g}}{\partial K_{r}}\right] \\
& -\eta_{1} E^{\varphi}\left(K_{r}\right)^{\prime} \frac{\partial^{2} f_{g}}{\partial K_{r}^{2}} .
\end{aligned}
$$

We see that it is very similar to the one found in Sect. 3. As in that case, we obtain that the form

$$
f_{g}=\eta_{1}\left(E^{r}, E^{\varphi}\right)\left[f_{0}\left(E^{r}, E^{\varphi}, K_{\varphi}\right)+K_{r} f_{1}\left(E^{r}, K_{\varphi}\right)\right],
$$

cancels the coefficients of $\left(K_{r}\right)^{\prime}$ and $\left(E^{\varphi}\right)^{\prime}$. In addition, the vanishing of the anomaly demands:

$$
\begin{aligned}
0= & \left(1-\frac{\eta_{3}}{\eta_{1}}\right) \frac{f_{1} E^{\varphi}}{2 E^{r}} \\
& +\frac{\partial f_{0}}{\partial K_{\varphi}}+(1-\omega) K_{r} \frac{\partial f_{1}}{\partial K_{\varphi}}-E^{\varphi} \frac{\partial f_{1}}{\partial E^{r}}
\end{aligned}
$$

which will be further analyzed below. On the other hand, for the anomaly $\mathcal{A}_{\mathcal{D}}^{D D}$ we find

$\mathcal{A}_{\mathcal{D}}^{D D} \propto \mathcal{A}_{\mathcal{D}}^{D H 2}-E^{\varphi} \frac{\partial \omega}{\partial E^{\varphi}}$,

which means that $\omega$ cannot depend on $E^{\varphi}$ as $\mathcal{A}_{\mathcal{D}}^{D H 2}$ must vanish on its own. In fact, the condition $\mathcal{A}_{\mathcal{D}}^{D H 2}=0$ reads explicitly,

$0=1-\omega-K_{r} \frac{\partial \omega}{\partial K_{r}}$

which is solved by

$\omega=1+\frac{\omega_{1}\left(E^{r}, K_{\varphi}\right)}{K_{r}}$.

We implement now conditions (A.6) and (A.10) in the anomaly $\mathcal{A}_{\mathcal{D}}^{D H 1}$ :

$$
\begin{aligned}
\mathcal{A}_{\mathcal{D}}^{D H 1}= & \frac{\left[\left(E^{r}\right)^{\prime}\right]^{2}}{8}\left(\frac{\partial \eta_{1}}{\partial E^{\varphi}} \eta_{3}-\eta_{1} \frac{\partial \eta_{3}}{\partial E^{\varphi}}\right. \\
& \left.+4 \eta_{1}^{2} \frac{E^{r}}{\left(E^{\varphi}\right)^{2}} \frac{\partial \omega_{1}}{\partial K_{\varphi}}\right)
\end{aligned}
$$

$$
\begin{aligned}
& +\frac{\eta_{1}^{2}}{\sqrt{E^{r}}}\left(f_{0}-\omega_{1} f_{1}-E^{\varphi} \frac{\partial f_{0}}{\partial E^{\varphi}}\right) \\
& +\frac{\left(E^{\varphi}\right)^{2}}{2}\left(\eta_{1} \frac{\partial \eta_{2}}{\partial E^{\varphi}}-\frac{\partial \eta_{1}}{\partial E^{\varphi}} \eta_{2}\right) .
\end{aligned}
$$

Without loss of generality, we set $\eta_{2}=\xi_{2}\left(E^{r}\right) \eta_{1}$ as in previous derivations, which annihilates the last term. Since there is no dependence of the free functions on radial derivatives, the vanishing of the above anomaly is then equivalent to the following two equations:

$0=\frac{\partial \eta_{1}}{\partial E^{\varphi}} \eta_{3}-\eta_{1} \frac{\partial \eta_{3}}{\partial E^{\varphi}}+4 \eta_{1}^{2} \frac{E^{r}}{\left(E^{\varphi}\right)^{2}} \frac{\partial \omega_{1}}{\partial K_{\varphi}}$,

$0=f_{0}-\omega_{1} f_{1}-E^{\varphi} \frac{\partial f_{0}}{\partial E^{\varphi}}$.

In the first equation, the only potential dependence on the angular component of the curvature $K_{\varphi}$ is contained in the function $\omega_{1}$. Therefore it must be at most linear in that variable, $\omega_{1}:=\xi_{d}\left(E^{r}\right)+K_{\varphi} \xi_{\omega}\left(E^{r}\right)$; otherwise this equation would not be satisfied. Taking this into account, the respective solutions of the last equations are.:

$\eta_{3}=\eta_{1}\left[\frac{\xi_{3}\left(E^{r}\right)}{\xi_{1}\left(E^{r}\right)}-4 \frac{E^{r}}{E^{\varphi}} \xi_{\omega}\left(E^{r}\right)\right]$,

$f_{0}=\omega_{1} f_{1}+E^{\varphi} f_{2}\left(E^{r}, K_{\varphi}\right)$.

Plugging these expressions into (A.7) leads to:

$0=\frac{\partial f_{2}}{\partial K_{\varphi}}+\frac{f_{1}}{2 E^{r}}\left(1-\frac{\xi_{3}}{\xi_{1}}+6 \frac{E^{r}}{E^{\varphi}} \xi_{\omega}\right)-\frac{\partial f_{1}}{\partial E^{r}}$.

Note that the only dependence of this equation on $E^{\varphi}$ is explicit, as none of the free functions depends on it. This means that either $f_{1}=0$ or $\xi_{\omega}=0$ should be obeyed. The first option is not of interest since it would further imply that $f_{2}$ is independent of $K_{\varphi}$ and one would then obtain that the function $f_{g}$ (and thus the modified Hamiltonian) would be completely independent of the curvature components $K_{r}$ and $K_{\varphi}$. As we are not considering singular solutions, we impose $\xi_{\omega}=0$. Finally, defining $h_{1}:=-\xi_{1} f_{1} /\left(2 \sqrt{E^{r}}\right)$ and $h_{2}:=-2 \sqrt{E^{r}} \xi_{1} f_{2}$, with $\xi_{1}=\xi_{1}\left(E^{r}\right)$, the last relation takes the same form as the anomaly equation (27).

After imposing all these conditions, the last anomaly $\mathcal{A}_{\mathcal{D}}^{D H 0}$ also vanishes and the modified diffeomorphism constraint reads,

$$
\begin{aligned}
& \widetilde{\mathcal{D}}_{g}=\Omega\left(E^{r}, E^{\varphi}, K_{r}, K_{\varphi}\right) \\
& \quad \times\left[\left(K_{\varphi}\right)^{\prime} E^{\varphi}-\left(K_{r}+\xi_{d}\left(E^{r}\right)\right)\left(E^{r}\right)^{\prime}\right] .
\end{aligned}
$$

The function $\xi_{d}\left(E^{r}\right)$, also present in the Hamiltonian constraint, can be absorbed performing the canonical transformation $\bar{K}_{r}=K_{r}+\xi_{d}$. In this way, the only modification to 
the diffeomorphism constraint is a global multiplicative function $\Omega$. As commented in the main body of the article, this kind of global multiplicative functions are trivially allowed in the constraints by the requirement of anomaly freedom.

Finally, the modified Hamiltonian takes the same form (28) as above (with the variable $\bar{K}_{r}$ instead of the initial $K_{r}$ ) and, therefore, we conclude that allowing for modifications to the vacuum diffeomorphism constraint does not involve additional freedom to include more corrections in the Hamiltonian constraint.

\section{Appendix B: Leading-order holonomic corrections in the anomaly-free semiclassical sector}

In this appendix, an approximate solution to the equation (27) will be found, which will provide the leading-order terms of the holonomy corrections in the pure vacuum model. Following the notation of the improved-dynamics scheme of loop quantum cosmology, we include a parameter $\bar{\mu}=\bar{\mu}\left(E^{r}\right)$ that accounts for the discreteness scale. Note that if the holonomy corrections $h_{1}$ and $h_{2}$ are scale-independent, both functions are allowed to be periodic in $K_{\varphi}$, as the last term in (27) drops out. In contrast, when one considers a length-dependent holonomy $h_{1}$, the additional requisite that $\partial h_{1} / \partial E^{r}$ is periodic in $K_{\varphi}$ must also be satisfied. For example, a sinusoidal function as $h_{1}=\sin \left(\bar{\mu} K_{\varphi}\right) / \bar{\mu}$, with $\bar{\mu}=\bar{\mu}\left(E^{r}\right)$, produces a term proportional to $K_{\varphi}$ on the right-hand side of (27), and thus $h_{2}$ can not be periodic in $K_{\varphi}$. However, as we are working in a semiclassical approach, we can focus on leadingorder holonomy corrections and try a power-series solution for $h_{1}$ and $h_{2}$. Let us assume that both holonomy corrections can be written as follows:

$h_{1}=\sum_{n=0}^{\infty} a_{n} \bar{\mu}^{n} K_{\varphi}^{n+1}$,

$h_{2}=\sum_{n=0}^{\infty} b_{n} \bar{\mu}^{n} K_{\varphi}^{n+2}$,

where the coefficients $a_{n}$ and $b_{n}$ are allowed to depend on $E^{r}$. Assuming now the form $\bar{\mu}\left(E^{r}\right)=\left(\Lambda^{2} / E^{r}\right)^{N / 2}$, with a constant $\Lambda$, the anomaly (27) provides the relation:

$\frac{n+2}{2} b_{n}=\left(\frac{\xi_{3}}{\xi_{1}}-2 E^{r} \frac{\partial \log \xi_{1}}{\partial E^{r}}-n N\right) a_{n}+2 E^{r} \frac{\partial a_{n}}{\partial E^{r}}$.

If we demand now constant values for the coefficients $a_{n}$ and $b_{n}$ (as one would expect if $\bar{\mu}$ retained all the scale dependence), and no inverse-triad corrections $\left(\xi_{1}=\xi_{3}=1\right)$, the last term in (B.20) drops out. In a sinusoidal approximations for $h_{1}$, only even coefficients would survive and therefore,

$b_{2 n}=-\frac{2 n-1}{n+1} a_{2 n}$,

where we have chosen $N=1$. For instance, if the usual ansatz $h_{1}=\sin \left(\bar{\mu} K_{\varphi}\right) / \bar{\mu}$ is chosen, the leading terms for the holonomy correction functions are explicitly given by,

$h_{1} \approx K_{\varphi}-\frac{1}{6} \frac{\Lambda^{2}}{E^{r}} K_{\varphi}^{3}$,

$h_{2} \approx K_{\varphi}^{2}+\frac{1}{12} \frac{\Lambda^{2}}{E^{r}} K_{\varphi}^{4}$

\section{References}

1. T. Thiemann, Modern Canonical Quantum General Relativity. Cambridge Monographs on Mathematical Physics (Cambridge University Press, Cambridge, 2007)

2. A. Ashtekar, P. Singh, Loop quantum cosmology: a status report. Class. Quantum Gravity 28, 213001 (2011)

3. I. Agullo, P. Singh, Loop quantum cosmology, in Loop Quantum Gravity: The First 30 Years, ed. by A. Ashtekar, J. Pullin (WSP, 2017), pp. 183-240

4. A. Ashtekar, Symmetry reduced loop quantum gravity: a bird's eye view. Int. J. Mod. Phys. D 25, 1642010 (2016)

5. A. Ashtekar, M. Bojowald, Quantum geometry and the Schwarzschild singularity. Class. Quantum Gravity 23, 391 (2006)

6. R. Gambini, J. Pullin, Black holes in loop quantum gravity: the complete spacetime. Phys. Rev. Lett. 101, 161301 (2008)

7. A. Corichi, P. Singh, Loop quantization of the Schwarzschild interior revisited. Class. Quantum Gravity 33, 055006 (2016)

8. A. Barrau, K. Martineau, F. Moulin, A status report on the phenomenology of black holes in loop quantum gravity: evaporation, tunneling to white holes, dark matter and gravitational waves. Universe 4, 102 (2018)

9. A. Ashtekar, J. Olmedo, P. Singh, Quantum extension of the Kruskal spacetime. Phys. Rev. D 98, 126003 (2018)

10. A. Ashtekar, J. Olmedo, P. Singh, Quantum transfiguration of Kruskal black holes. Phys. Rev. Lett. 121, 241301 (2018)

11. H. Morales-Tecotl, S. Rastgoo, J. Ruelas, Effective dynamics of the Schwarzschild black hole interior with inverse-triad corrections Ann. Phys. 426, 168401 (2021). https://doi.org/10.1016/j. aop.2021.168401

12. N. Bodendorfer, F.M. Mele, J. Münch, Effective quantum extended spacetime of polymer Schwarzschild black hole. Class. Quantum Gravity 36, 195015 (2019)

13. M. Bouhmadi-López, S. Brahma, C.-Y. Chen, P. Chen, D. Yeom, A consistent model of non-singular Schwarzschild black hole in loop quantum gravity and its quasinormal modes. JCAP 07, 066 (2020)

14. J. Olmedo, S. Saini, P. Singh, From black holes to white holes: a quantum-gravitational symmetric bounce. Class. Quantum Gravity 34, 225011 (2017)

15. M. Martín-Benito, L .J. Garay, G .A.Mena Marugán, Hybrid quantum Gowdy cosmology: combining loop and Fock quantizations. Phys. Rev. D 78, 083516 (2008)

16. M. Fernández-Méndez, GA Mena Marugán, J. Olmedo, Hybrid quantization of an inflationary universe. Phys. Rev. D 86, 024003 (2012)

17. M. Fernández-Méndez, GA Mena Marugán, J. Olmedo, Hybrid quantization of an inflationary model: the flat case. Phys. Rev. D 88, 044013 (2013) 
18. L. Castelló, M. Fernández-Méndez, GA Mena Marugán, J. Olmedo, Cosmological perturbations in hybrid loop quantum cosmology: Mukhanov-Sasaki variables. Phys. Rev. D 90, 064015 (2014)

19. L. Castelló Gomar, G.A. Mena Marugán, M. Martín-Benito, Quantum corrections to the Mukhanov-Sasaki equations. Phys. Rev. D 93, 104025 (2016)

20. I. Agullo, A. Ashtekar, W. Nelson, Extension of the quantum theory of cosmological perturbations to the Planck era. Phys. Rev. D 87, 043507 (2013)

21. I. Agullo, A. Ashtekar, W. Nelson, The pre-inflationary dynamics of loop quantum cosmology: confronting quantum gravity with observations. Class. Quantum Gravity 30, 085014 (2013)

22. I. Agullo, A. Ashtekar, B. Gupt, Phenomenology with fluctuating quantum geometries in loop quantum cosmology. Class. Quantum Gravity 34, 074003 (2017)

23. M. Bojowald, Non-covariance of the dressed-metric approach in loop quantum cosmology. Phys. Rev. D 102, 023532 (2020)

24. M. Bojowald, G.M. Hossain, M. Kagan, S. Shankaranarayanan, Anomaly freedom in perturbative loop quantum gravity. Phys. Rev. D 78, 063547 (2008)

25. M. Bojowald, M. Kagan, P. Singh, H.H. Hernández, A. Skirzewski, Hamiltonian cosmological perturbation theory with loop quantum gravity corrections. Phys. Rev. D 74, 123512 (2006)

26. M. Bojowald, G.M. Hossain, M. Kagan, S. Shankaranarayanan, Gauge-invariant cosmological perturbation equations with corrections from loop quantum gravity. Phys. Rev. D 79, 043505 (2009)

27. T. Cailleteau, J. Mielczarek, A. Barrau, J. Grain, Anomaly-free scalar perturbations with holonomy corrections in loop quantum cosmology. Class. Quantum Gravity 29, 095010 (2012)

28. T. Cailleteau, A. Barrau, F. Vidotto, J. Grain, Consistency of holonomy-corrected scalar, vector and tensor perturbations in loop quantum cosmology. Phys. Rev. D 86, 087301 (2012)

29. Y. Han, M. Liu, Anomaly-free cosmological perturbations with generalised holonomy correction in loop quantum cosmology. Class. Quantum Gravity 35, 105017 (2018)

30. R. Gambini, J. Pullin, Loop quantization of the Schwarzschild black hole. Phys. Rev. Lett. 110, 211301 (2013)

31. R. Gambini, J. Pullin, Hawking radiation from a spherical loop quantum gravity black hole. Class. Quantum Gravity 31, 115003 (2014)

32. M. Bojowald, S. Brahma, J.D. Reyes, Covariance in models of loop quantum gravity: spherical symmetry. Phys. Rev. D 92, 045043 (2015)

33. M. Bojowald, J. Reyes, R. Tibrewala, Nonmarginal LemaitreTolman-Bondi-like models with inverse-triad corrections from loop quantum gravity. Phys. Rev. D 80, 084002 (2009)

34. J.D. Reyes, Spherically symmetric loop quantum gravity: connections to two-dimensional models and applications to gravitational collapse. Ph.D. thesis, Pennsylvania State University (2009)

35. R. Tibrewala, Spherically symmetric Einstein-Maxwell theory and loop quantum gravity corrections. Class. Quantum Gravity 29, $235012(2012)$
36. M. Bojowald, S. Brahma, U. Buyukcam, F. D'Ambrosio, Hypersurface-deformation algebroids and effective spacetime models. Phys. Rev. D 94, 104032 (2016)

37. M. Bojowald, S. Brahma, D.H. Yeom, Effective line elements and black-hole models in canonical loop quantum gravity. Phys. Rev. D 98, 046015 (2018)

38. D. Arruga, J.B. Achour, K. Noui, Deformed general relativity and quantum black holes interior. Universe 6, 39 (2020)

39. M. Bojowald, Black-hole models in loop quantum gravity. Universe 6, 125 (2020)

40. T. Thiemann, QSD 5: quantum gravity as the natural regulator of matter quantum field theories. Class. Quantum Gravity 15, 1281 (1998)

41. M. Bojowald, The inverse scale factor in isotropic quantum geometry. Phys. Rev. D 64, 084018 (2001)

42. A. Ashtekar, T. Pawlowski, P. Singh, Quantum nature of the Big Bang: improved dynamics. Phys. Rev. D 74, 084003 (2006)

43. V. Belinsky, I. Khalatnikov, E. Lifshitz, Oscillatory approach to a singular point in the relativistic cosmology. Adv. Phys. 19, 525 (1970)

44. W. Kamiñski, J. Lewandowski, M. Bobienski, Background independent quantizations: the scalar field I. Class. Quantum Gravity 23, 2761 (2005)

45. W. Kamiński, J. Lewandowski, A. Okołów, Background independent quantizations: the scalar field II. Class. Quantum Gravity 23, 5547 (2006)

46. F. Benítez, R. Gambini, L. Lehner, S. Liebling, J. Pullin, Critical collapse of a scalar field in semiclassical loop quantum gravity. Phys. Rev. Lett. 124, 071301 (2020)

47. J.B. Achour, Towards self-dual loop quantum gravity. Ph.D. thesis, Paris U. IV (2015)

48. J.B. Achour, S. Brahma, J. Grain, A. Marciano, A new look at scalar perturbations in loop quantum cosmology: (un)deformed algebra approach using self-dual variables (2016). arXiv:1610.07467 [grqc]

49. J.B. Achour, S. Brahma, A. Marciano, Spherically symmetric sector of self-dual Ashtekar gravity coupled to matter: anomaly-free algebra of constraints with holonomy corrections. Phys. Rev. D 96, 026002 (2017)

50. J.B. Achour, S. Brahma, Covariance in self-dual inhomogeneous models of effective quantum geometry: spherical symmetry and Gowdy systems. Phys. Rev. D 97, 126003 (2018)

51. M. Bojowald, G.M. Paily, J.D. Reyes, Discreteness corrections and higher spatial derivatives in effective canonical quantum gravity. Phys. Rev. D 90, 025025 (2014) 